\title{
SLC17A9 Is a Novel Prognostic Biomarker Correlated With Immune Infiltrates in Human Lung Adenocarcinoma and Lung Squamous Cell Carcinoma
}

\section{Yan Gao}

Hubei University of Medicine

Yi-Jia Chen

Hubei University of Medicine

Fuyan Li

Hubei University of Medicine

Ruimin Wu

Hubei University of Medicine

Daobing Zeng

Hubei University of Medicine

\section{Fan Tan}

Hubei University of Medicine

Xusheng Liu

Hubei University of Medicine

Xiaohui Li

Hubei University of Medicine

Yang Li

Hubei University of Medicine

Shan-Chun Su

Hubei University of Medicine

Jianwei Yang

Hubei University of Medicine

Lumeng Zhou

Hubei University of Medicine

Changbin Ke

Hubei University of Medicine

Zhijun Pei ( $\sigma$ pzjzml1980@taihehospital.com )

Hubei University of Medicine

\section{Research Article}

Keywords: SLC17A9, prognosis, lung adenocarcinoma, lung squamous cell carcinoma, immune infiltrates 
Posted Date: June 1st, 2021

DOI: https://doi.org/10.21203/rs.3.rs-517084/v1

License: (c) (i) This work is licensed under a Creative Commons Attribution 4.0 International License. Read Full License 


\section{Abstract \\ Background}

Overexpression of vesicular nucleotide transporter (SLC17A9) has been found in different types of cancers. Nonetheless, little is known about its influence on lung cancers including human lung adenocarcinoma (LUAD) and lung squamous cell carcinoma (LUSC).

\section{Methods}

Integrative analysis of SLC17A9 and other solute carrier family 17 genes (SLC17A1-8) was performed in patients with LUAD and LUSC based on The Cancer Genome Atlas database. Real-time PCR, western blots, MTS assay, EdU assay, ATP production assays and cell cycle analysis were applied to determine the effect and mechanism of SLC17A9 knockdown in LUAD cells.

\section{Results}

Compared with normal tissue, two SLC17 genes (SLC17A5 and SLC17A9) exhibited a distinctly different expression pattern in LUAD and LUSC. The expression of SLC17A3/7/8/9 expression was significantly correlated with worse overall survival $(p<0.05)$ in LUAD. Conversely, SLC17A1/2/4/6/9 expression was correlated with poorer OS $(p<0.05)$ in LUSC. ROC analysis suggested that the area under the curve of most SLC17 family genes was higher than 0.5 . Meanwhile, multiple types of genetic alterations in SLC17 family genes were observed in tumor samples. Gene set enrichment analysis GSEA and protein-protein interaction results revealed that oncogenic signaling pathways and biological regulation, metabolic process, hallmark of myc targets, DNA repair, coagulation and complement were linked to SLC17A9 upregulation. Moreover, SLC17A9 knockdown significantly inhibited cell proliferation and ATP levels by affecting Myc, MFN2, STAT3, Cytochrome C and P2X1 expression in A549 cells. Specifically, SLC17A9 expression correlated negatively with overall survival and positively with most LUSC immune cells. SLC17A9 expression has correlations with infiltrating levels of B cells, CD4 + T cells, M1 macrophages, natural killer cells, Th1, Th2, Tfh, Th17 and Treg cells, as well as PD-1, CTLA4, and LAG3 of T cell exhaustion in LUAD.

\section{Conclusions}

Together, SLC17A9 could potentially serve as a prognostic biomarker correlated with immune infiltrates in LUAD and LUSC.

\section{Introduction}

Lung cancer is the leading cause of cancer death in the world, and non-small cell lung cancer (NSCLC) accounts for approximately $85 \%$ of lung cancer cases [1]. The 5-year survival rate of NSCLC is much higher in the early stage, but only $4.5 \%$ for metastatic disease [2]. Lung adenocarcinoma (LUAD) and lung squamous 
cell carcinoma (LUSC) are the most prevalent histopathological subtypes of NSCLC. Nevertheless, poor prognosis remains a big clinical challenge, and identifying early diagnostic biomarkers and treatment options is crucial for LUAD and LUSC [2]. Combined with bioinformatics analysis, our previous study uncovered a new molecular target named nucleophosmin 1 that could help improve diagnostic accuracy in LUAD cases [3]. In the past decades, large-scale genomic studies have revealed molecular features and improved our understanding of the underlying mechanisms of many tumour types, which might explain the associations in survival among patients with different pathological staging [4-7]. In addition, an increasing number of studies have found that immunotherapy targeting the tumor microenvironment (TME), including cancer cells and innate and adaptive immune cells, is a strategy strongly pursued against human cancers [8]. Cytotoxic $T$ lymphocyte associated antigen 4 (CTLA4), programmed death-1 (PD-1) and programmed death ligand-1 (PDL1) in TME serve as immune checkpoint proteins that inhibit the T-cell mediated immune responses in NSCLC $[9,10]$. Therefore, comprehensive multi-platform analyses may be helpful for prognostics and screening of biomarkers involved in tumour progression in the early stage of NSCLC.

Solute carrier family 17 (SLC17) consists of membrane transport proteins, which are responsible for the transport of organic anions. To date, nine transmembrane segment transporters (SLC17A1-9) have been identified, including type I phosphate transporters (SLC17A1-4), vesicular glutamate transporters (SLC17A68), a lysosomal acidic sugar transporter (sialin; SLC17A5) [11]. The most recently described vesicular nucleotide transporter, also called SLC17A9, mediates liposomal ATP accumulation and could regulate cell viability, cancer progression [12-14] and immune activations [15]. However, few studies have evaluated the expression of SLC17As in NSCLC, and little is known regarding the underlying functions of SLC17A9 in NSCLC, which still require further investigation.

In the present study, we analyzed the mRNA expression of all nine subsets of SLC17 using data from The Cancer Genome Atlas (TCGA) database, and investigated its associations with survival outcomes in LUAD and LUSC, that imply that several SLC17As including SLC17A9 may have prognostic value in NSCLC. To comprehensively determine the mechanism by which SL17A9 expression influences the prognosis, genetic alteration and dysregulated function in NSCLC patients, Gene ontology (GO), Kyoto Encyclopedia of Genes and Genomes (KEGG) pathways enrichment, Gene Set Enrichment Analysis (GSEA), as well as protein-protein interaction (PPI) network of co-expressed genes were performed. In addition, in vitro studies revealed that SLC17A9 knockdown had a marked impact on cell proliferation, regulated by oncogenes and cancerassociated pathways in NSCLC. Finally, we also tried to explore the correlation between differential SLC17A9 expression and infiltrating immune cells in TCGA-LUAD and LUSC datasets. These findings provide new evidence elucidating the anti-tumor role of SLC17A9 and a previously unknown molecular biomarker involved in NSCLC tumor progression.

\section{Materials And Methods}

\section{Expression analysis by TCGA and GEO database}

The mRNA expression profiles (HTSeq FPKM and HTSeq counts) and associated clinical data for 107 normal lung tissues samples, as well as 510 LUAD and 546 LUSC patients were downloaded from TCGA database (https://portal.gdc.cancer.gov/). The expression profiling of nine SL17A family genes was plotted in the 
ggplot2 R (https://github.com/tidyverse/ggplot2)[16]. To analyze the differences in SLC17A9 expression between NSCLC and non-cancer samples, the expression profiles of GSE18842, GSE33532, GSE74706, GSE116959, and GSE4127 were downloaded from the Gene Expression Omnibus (GEO) database. The GPL570 platform was used to obtain both GSE18842 and GSE33532. The GSE74706, GSE116959 and GSE4127 comes from GPL13497, GPL17077 and GPL96 platform, respectively.

\section{Survival prognosis analysis in Kaplan-Meier Plot and GEPIA2}

Kaplan-Meier analysis was conducted to evaluate gene-associated hazard ratios (HRs) for overall survival (OS) based on RNA-seq data and genechip data from GEO, CEA and TCGA database. In the study, KaplanMeier plots (http://kmplot.com/ analysis/) [17] and R packages were used to analyze the correlation between gene expression and OS rates in LUAD and LUSC patients.

The survival map of "hub" genes was drawn by GEPIA2 (Gene Expression Profiling Interactive Analysis) web server. The receiver operating characteristic (ROC) curve was used to calculate the area under curve (AUC), to evaluate the prognostic or predictive accuracy. The "pROC" of R package was used to perform the ROC curve analysis with the clinicopathological parameters of TCGA lung cancer.

\section{Mutations and copy number alterations in cBioPortal analysis}

To find the genomic profiles of nine SL17A family members, the location and frequency of SL17A alterations (amplifications, deep deletions, missense mutations and truncating mutations) and copy number variance data were retrieved from TCGA lung cancer datasets using the online resource cBiopPortal (www.cbioportal.org)[18].

\section{Screening of SL17A9 co-expressed genes via LinkedOmics database and functional enrichment analysis}

The LinkedOmics database (http://www.linkedomics.org/login.php) is a web-based platform for analyzing all 32 TCGA cancer-associated multi-dimensional datasets[19]. SL17A9 co-expression in LUAD and LUSC was compared statistically using Pearson's correlation coefficient, presented in heat maps. Subsequently, the GO: BP (biological process), CC (cellular component), MF (molecular function) and KEGG pathway analyses for co-expressed genes were signed and ranked.

\section{Gene Set Enrichment Analysis (GSEA)}

In the present study, Gene Set Enrichment Analysis (GSEA) was conducted to identify significant differences between low and high expression of SLC17A9 groups in NSCLC using GEO matrix of the GSE18842. The H hallmark gene sets were downloaded from the molecular signatures database (MSigDB v7.2). The NES and nominal $p$ value for the GSEA test were calculated by permutation number set at 1000 . Enriched gene sets were selected for FDR $<0.05$ and NES $>1.50$.

\section{PPI network construction}

Search Tool for the Retrieval of Interacting Genes/Proteins (STRING) 11.0 (https://string-db.org/) is a database of known and predicted protein interactions that contains the direct and indirect protein 
associations[20]. SLC17A9 was imported into the STRING database, selecting those with a combined score > 0.4. Next, the CytoHubba plugin in Cytoscape was employed to calculate the degree of each protein node and identify 'hub' genes. The top 10 genes were defined as 'hub' genes.

\section{Estimation of Immune Cell Type Fractions}

The Tumor Immune Estimation Resource (TIMER, https://cistrome.shinyapps.io/ timer/) database includes 10,897 samples across 32 cancer types from TCGA to illustrate correlation between a pair of genes using the Spearman correlation coefficient and to estimate statistical significance[21]. It was utilized to estimate the abundance of immune infiltrates and analyze the correlation between SLC17A9 expression levels and the signatures and gene markers of tumor-infiltrating immune cells, as well as the tumor purity in LUAD and LUSC. In addition, the xCell, pheatmap, gene set variation analysis (GSVA), and ssGSEA R packages were utilized for reliable immune cell type enrichment analysis. Next, the expression of immune checkpointrelevant genes including SIGLEC15, ID01, CD274, HAVCR2, PDCD1, CTLA4, LAG3, and PDCD1LG2 were extracted from TCGA database. The ggplot2 and pheatmap R packages were utilized to explore the relationship between SLC17A9 and immune checkpoints in LUAD and LUSC.

\section{Cell culture and treatment}

Human LUAD cell lines A549 were purchased from ATCC and cultured in high glucose DMEM (HyClone) supplemented with $10 \%$ fetal bovine serum (Gibco). The small interfering RNA (siRNA) targeting human SLC17A9 was synthesized by GenePharma and transfected into the cells using Lipofectamine 8000 reagent (Beyotime). Non-specific siRNA was used as negative controls. The oligonucleotide sequences of the siRNA were displayed as follows:

siSLC17A9, Sense 5'-UUUCUGUUAACAUCCAGGATT-3'; Antisense 5'- UCCUGGAUGUUAACAGAAAUG-3'. siNC, Sense 5'-ACGUGACACGUUCGGAGAA-3'; Antisense 5'-UCUUCUCCGAACGUGUCACGU-3'.

\section{RNA isolation and real-time quantitative PCR analysis}

Total RNA was extracted using TRIzol reagent (Invitrogen). For mRNA analysis, cDNA was synthesized using the TaqMan Reverse Transcription Reagents kit (TaKaRa). Real-time quantitative PCR analysis was performed with different primer sequences using SYBR Green Master Mix (TaKaRa). The primers used for RTPCR were shown in Table 1.

\section{Table 1 Primer sequences for real time RT-PCR}




\begin{tabular}{|lll|}
\hline Target & Forward & Reverse \\
\hline ACTB & TCTTCCAGCCTTCCTTCCT & AGCACTGTGTTGGCGTACAG \\
SLC17A9 & AGGGGTTTACTTCCCTGCC & GTCAGCAGCGTCCCAAACT \\
P2RX1 & CTACAATGACCACCATCGGCTCTG & CTTAGGCAGGATGTGAAGCAGCAG \\
MYC & CGCCTCTTGACATTCTCCTC & GGACTATCCTGCTGCCAAGA \\
Cytochrome C & ATTGGCGGCTGTGTAAGAGT & AAGTGTTCCCAGTGCCACA \\
MFN2 & AGCCCTCGGTAAGGAGAAAG & GTGATCAATGCCATGCTCTG \\
STAT3 & TCGGCTAGAAAACTGGATAACG & TGCAACTCCTCCAGTTTCTTAA \\
\hline
\end{tabular}

\section{Western blotting}

The cells proteins were isolated, quantified, and separated via SDS-PAGE. Western blotting was performed as described previously [22]. Primary antibodies included the rabbit $\beta$-Actin antibody (Cell Signaling Technology), and the SLC17A9 antibody (Abcam). Anti-rabbit secondary antibodies (Cell Signaling Technology) were used at a dilution of 1:6000.

\section{Cell proliferation detection via MTS and EdU assays}

The cell viability and growth rate were assessed at 48,72 and 96 hours after transfection with siRNA, using MTS Assay Kits (Promega) according to the manufacturer's instructions. A standard immunofluorescence assay protocol was performed using the BeyoClick ${ }^{\text {TM }}$ EdU-488 assay kit (Beyotime) following the manufacturer's instructions. In brief, following incubation with $10 \mu \mathrm{M}$ Edu for $2 \mathrm{~h}$, the indicated cells were fixed, permeabilized and stained with the Click Additive Solution and DAPI. The images were acquired using a confocal laser-scanning microscope. The density of EdU-positive nuclei was calculated for cell proliferation analysis.

\section{ATP detection assay}

An ATP bioluminescent assay kit (Beyotime, China) was used to measure intracellular ATP levels according to manufacturer's instructions. A549 cells were seeded in 6-well plates and collected at 72 hours after transfection with siRNA. The cellular ATP was extracted using appropriate buffer, mixed with ATP detection solution containing luciferase, and the bioluminescence was measured using a luminescence plate reader. The total ATP levels were expressed as $\mathrm{pmol} / \mu \mathrm{g}$ protein.

\section{Cell cycle analysis}

Briefly, cells were collected at 72 hours after transfection with siRNA. After fixation with $70 \%$ ice cold ethanol at $4^{\circ} \mathrm{C}$ for 12 hours, cells were stained with propidium iodide $(50 \mu \mathrm{g} / \mathrm{ml})$ for 30 minutes, and then the flow FACS Gallios Flow Cytometer (BD Biosciences) was applied to detect cell cycle changes.

\section{Statistical analysis}


SPSS version 24.0 was used for data analyses. The $\chi 2$ square test and Spearman correlation test were performed to evaluate the relationship between SLC17A expression and the clinical features of tumor progression. The R packages implemented by $\mathrm{R}$ foundation for statistical computing (version 4.0.3) are listed below: ggplot2, survival, survminer, pROC, xCell, GSVA, sSGSEA, and pheatmap. Results are expressed as the mean \pm standard deviation. Statistical differences between groups were analyzed via student's t-test or oneway analysis of variance using SPSS version 24.0 statistical software (SPSS, Inc., USA). A two-tailed P< 0.05 was considered statistically significant.

\section{Results}

\section{Expression of SLC17A family members and the prognostic significance in LUAD and LUSC}

To explore the clinical relevance of the SLC17A family in NSCLC, the association between MRNA expression levels of nine SLC17As and cancer prognosis was determined in the human LUAD and LUSC cases in TCGA database. Noticeably, as shown in Fig. $1 A$ and B, compared with normal lung tissues, only SLC17A9 mRNA expression was significantly upregulated $(p<0.001)$, while SLC17A5 expression was significantly lower in tumour tissues in both LUAD and LUSC samples. Conversely, SLC17A1/2/3/4//6/7/8 expression was slightly decreased (fold change $<1.2$ ) in tumour tissues.

Online analysis tools and R software were used to analyze the efficacy of nine SLC17As for prognosis in LUAD and LUSC patients. Data from Kaplan-Meier plotter indicated that SLC17A3/7/8/9 expression correlated with poorer OS in 513 LUAD patients (Fig. 1C), and SLC17A1/2/4/6/9 expression was correlated with poorer OS in 501 LUSC patients (Fig. 1D). Other members of this protein family did not show any significant correlation with survival rate. Univariate logistic regression analysis revealed that only SLC17A9 could serve as an OS marker in LUAD [HR=0.684 (0.512-0.914), $P=0.01]$ independent of clinicopathological parameters (Table 2).

Table 2 Univariate Cox regression analysis of SLC17As and clinical-pathological characteristics in LUAD and LUSC patients of TCGA dataset 


\begin{tabular}{|c|c|c|c|c|c|c|}
\hline \multirow[t]{2}{*}{ Characteristics } & \multicolumn{3}{|l|}{ LUAD } & \multicolumn{3}{|l|}{ LUSC } \\
\hline & $\begin{array}{l}\text { Total } \\
(\mathrm{N})\end{array}$ & $\mathrm{HR}(95 \% \mathrm{Cl})$ & $P$ value & $\begin{array}{l}\text { Total } \\
(\mathrm{N})\end{array}$ & $\begin{array}{l}\mathrm{HR}(95 \% \\
\mathrm{Cl})\end{array}$ & $\begin{array}{l}\mathrm{P} \\
\text { value }\end{array}$ \\
\hline Gender (Male vs. Female) & 526 & $\begin{array}{l}1.070 \\
(0.803- \\
1.426)\end{array}$ & 0.642 & 496 & $\begin{array}{l}1.211 \\
(0.879- \\
1.669)\end{array}$ & 0.241 \\
\hline Age (>65 vs. $<=65)$ & 516 & $\begin{array}{l}1.223 \\
(0.916- \\
1.635)\end{array}$ & 0.172 & 490 & $\begin{array}{l}1.279 \\
(0.960- \\
1.704)\end{array}$ & $0.093^{*}$ \\
\hline T stage (T2\&T3\&T4 vs. T1) & 523 & $\begin{array}{l}1.728 \\
(1.229- \\
2.431)\end{array}$ & $0.002^{\star}$ & 496 & $\begin{array}{l}1.377 \\
(0.984- \\
1.926)\end{array}$ & $0.062^{\star}$ \\
\hline N stage (N1\&N2\&N3 vs. N0) & 510 & $\begin{array}{l}2.601 \\
(1.944- \\
3.480)\end{array}$ & $<0.001^{*}$ & 490 & $\begin{array}{l}1.151 \\
(0.869- \\
1.523)\end{array}$ & 0.327 \\
\hline M stage (M1 vs. M0) & 377 & $\begin{array}{l}2.136 \\
(1.248- \\
3.653)\end{array}$ & $0.006^{\star}$ & 415 & $\begin{array}{l}3.112 \\
(1.272- \\
7.616)\end{array}$ & $0.013^{\star}$ \\
\hline $\begin{array}{l}\text { Pathologic stage (Stage III\& Stage IV } \\
\text { vs. Stage I\&Stage II) }\end{array}$ & 518 & $\begin{array}{l}2.664 \\
(1.960- \\
3.621)\end{array}$ & $<0.001 *$ & 492 & $\begin{array}{l}1.321 \\
(1.006- \\
1.735)\end{array}$ & $0.046^{*}$ \\
\hline SLC17A3 (High vs. Low) & 526 & $\begin{array}{l}0.832 \\
(0.623- \\
1.112)\end{array}$ & 0.214 & & & \\
\hline SLC17A5 (High vs. Low) & 526 & $\begin{array}{l}0.912 \\
(0.684- \\
1.216)\end{array}$ & 0.530 & 496 & $\begin{array}{l}1.124 \\
(0.858- \\
1.474)\end{array}$ & 0.396 \\
\hline SLC17A7 (High vs. Low) & 526 & $\begin{array}{l}1.203 \\
(0.902- \\
1.605)\end{array}$ & 0.208 & 496 & $\begin{array}{l}0.865 \\
(0.660- \\
1.134)\end{array}$ & 0.295 \\
\hline SLC17A8 (High vs. Low) & 526 & $\begin{array}{l}0.817 \\
(0.613- \\
1.090)\end{array}$ & 0.169 & 496 & $\begin{array}{l}1.073 \\
(0.818- \\
1.407)\end{array}$ & 0.611 \\
\hline SLC17A9 (High vs. Low) & 526 & $\begin{array}{l}0.684 \\
(0.512- \\
0.914)\end{array}$ & $0.010 *$ & 496 & $\begin{array}{l}1.156 \\
(0.882- \\
1.515)\end{array}$ & 0.295 \\
\hline
\end{tabular}

$\mathrm{Cl}$, confidence interval; LUAD, lung adenocarcinoma; LUSC, lung squamous cell carcinoma; HR, Hazard ratio. $* \mathrm{P}<0.05$

To further analyze the diagnostic value of SLC17A family genes from NSCLC patients in TCGA database, ROC curve analysis of the predicted efficiency was performed. It suggested that most of these genes had diagnostic value with corresponding AUC $(>0.5)$ for distinguishing NSCLC patients from healthy individuals. As Fig. 2A shows, SLC17A3 (0.750), SLC17A8 (0.766) and SLC17A9 (0.893) showed a moderate value (AUC $>0.7)$ for distinguishing LUAD patients. Conversely, SLC17A1 (0.701), SLC17A3 (0.932), SLC17A5 (0.897), 
SLC17A8 (0.811) and SLC17A9 (0.723) showed a moderate value (AUC >0.7) for LUSC patients (Fig. 2B). These results suggested that SLC17As might play important roles in the development of NSCLC.

\section{Genetic mutations in SLC17As in LUAD and LUSC}

We then tried to investigate the genetic alteration features in SLC17As of LUAD and LUSC using the cBioPortal database. The results showed the frequency of classification of genomic alterations, such as amplification, mutation, deep deletion, and multiple alterations. The genetic alteration percentages of SLC17A family genes for LUAD (Fig. 3A) and LUSC (Fig. 3B) varied from 1.1\% to $4.0 \%$ for individual genes. Meanwhile, there was no amplification of SLC17A8 in LUSC and LUAD. Fig.3C summarizes the details regarding the missense and truncating mutations in LUAD and LUSC. To predict the functional impact scores of all missense mutations identified in SLC17As, we used the Mutation Assessor from the cBioPortal cancer database. The mutation assessor score of R140L and W216R reached 3.5 (high impact on protein function) in SLC17A1. Other missense mutation sites with high score, including SLC17A4 (G211W, L114V and G448V), SLC17A6 (G225E), SLC17A8 (M103I, R444C and P175L) were also obtained, suggesting that they would be important regions for its function.

\section{Upregulation of SLC17A9 in NSCLC correlated with clinicopathological features}

Overexpression of SLC17A9 had the most relevant prognostic and diagnostic value in both LUAD and LUSC. Therefore, SLC17A9 was selected as a target of interest in the following research. To further confirm the expression features of SLC17A9 in NSCLC, we evaluated SLC17A9 expression in NSCLC using TCGA and five independent GEO datasets with microarray platforms. We also found that significantly higher SLC17A9 mRNA expression in LUAD, LUSC and NSCLC than in normal tissues $(P<0.05)$ (Fig. 4 A-E). As shown in Fig. 4F, SLC17A9 mRNA expression was also abundant in LUAD and LUSC cell lines, especially higher in LU65 and RERF-LC-AI (GSE4127). To determine the clinical relevance of SLC17A9 in NSCLC, we divided clinical LUAD and LUSC patients in TCGA database into low and high expression groups based on the median expression value of SLC17A9. The relationship between SLC17A9 expression and clinicopathological parameters of the LUAD patients $(n=535)$ and LUSC patients $(n=502)$ from TCGA database are displayed in Table 3. SLC17A9 overexpression was signifcantly correlated with OS in LUAD patients, but not with age, gender and stages ( $P>0.05)$.

Table 3 The association between SLC17A9 mRNA levels and clinical-pathological characteristics of LUAD and LUSC patients in TCGA dataset 


\begin{tabular}{|c|c|c|c|c|c|c|}
\hline \multirow[t]{2}{*}{ Characteristic } & \multicolumn{3}{|l|}{ LUAD } & \multicolumn{3}{|l|}{ LUSC } \\
\hline & Low & High & $p$ & Low & High & $p$ \\
\hline$n$ & 267 & 268 & & 251 & 251 & \\
\hline Age, meidan (IQR) & $67(60,72)$ & $65(58,72)$ & 0.294 & $67(62,73)$ & $69(62,74)$ & 0.258 \\
\hline Gender, n (\%) & & & 0.968 & & & 0.839 \\
\hline Female & $142(26.5 \%)$ & $144(26.9 \%)$ & & $64(12.7 \%)$ & $67(13.3 \%)$ & \\
\hline Male & $125(23.4 \%)$ & $124(23.2 \%)$ & & $187(37.3 \%)$ & $184(36.7 \%)$ & \\
\hline Age, n (\%) & & & 0.656 & & & 0.438 \\
\hline$<=65$ & $122(23.6 \%)$ & $133(25.8 \%)$ & & $100(20.3 \%)$ & $91(18.5 \%)$ & \\
\hline$>65$ & $131(25.4 \%)$ & $130(25.2 \%)$ & & $146(29.6 \%)$ & $156(31.6 \%)$ & \\
\hline T stage, $\mathrm{n}(\%)$ & & & 0.947 & & & 0.17 \\
\hline $\mathrm{T} 1,2$ & $230(43.3 \%)$ & $234(43.9 \%)$ & & $210(41.9 \%)$ & $198(39.5 \%)$ & \\
\hline Т3,4 & $34(6.4 \%)$ & $34(6.4 \%)$ & & $41(8.2 \%)$ & $53(10.6 \%)$ & \\
\hline $\mathrm{N}$ stage, $\mathrm{n}(\%)$ & & & 0.386 & & & 0.861 \\
\hline NO & $165(31.8 \%)$ & $183(35.3 \%)$ & & $161(32.5 \%)$ & $159(32.1 \%)$ & \\
\hline N1-3 & $88(17.0 \%)$ & $83(16.0 \%)$ & & $90(18.1 \%)$ & $86(17.3 \%)$ & \\
\hline M stage, n (\%) & & & 0.142 & & & 0.721 \\
\hline M0 & $183(47.4 \%)$ & $178(46.1 \%)$ & & 209 (49.9\%) & $203(48.4 \%)$ & \\
\hline M1 & $17(4.4 \%)$ & $8(2.1 \%)$ & & $3(0.7 \%)$ & $4(1 \%)$ & \\
\hline pStage, n (\%) & & & 0.704 & & & 0.474 \\
\hline Stage I & $144(27.3 \%)$ & $150(28.5 \%)$ & & $126(25.3 \%)$ & $119(23.9 \%)$ & \\
\hline Stage II-IV & $118(22.4 \%)$ & $115(21.8 \%)$ & & $122(24.5 \%)$ & $131(26.3 \%)$ & \\
\hline OS event, n (\%) & & & $0.005^{\star}$ & & & 0.417 \\
\hline Alive & 155 (29\%) & $188(35.1 \%)$ & & 138 (27.5\%) & $148(29.5 \%)$ & \\
\hline Dead & $112(20.9 \%)$ & $80(15 \%)$ & & 113 (22.5\%) & $103(20.5 \%)$ & \\
\hline
\end{tabular}

Kaplan-Meier survival curve analysis of LUAD and LUSC patients revealed that low mRNA expression of SLC17A9 was significantly associated with worse OS in LUAD based on RNA-seq data, while higher SLC17A9 expression from GeneChip data and TCGA database was positively correlated with poorer prognosis (log-rank test $\mathrm{P}<0.05$; Fig $4 \mathrm{G}$ and I). Conversely, higher SLC17A9 expression in LUSC was correlated with worse OS in LUAD, while SLC17A9 expression from GeneChip data was not significantly related to OS $(p=0.23$, Fig. $4 \mathrm{H}$ and J). Hence, we selected SLC17A9 as the target of our research. 


\section{Co-expressed genes and underlying mechanisms of SLC17A9 in LUAD and LUSC}

Gene co-expression analyses could reflect functional relationship, thus we examined genes co-expressed with SLC17A9 in the LUAD and LUSC transcriptional data from TCGA database. Fig. 5A showed the heat map of the top 10 positively and top 10 negatively co-expressed genes with SLC17A9. We compared the list of genes co-expressed with SLC17A9 between LUAD and LUSC, and found that more than 3,674 co-expressed genes were significantly correlated with SLC17A9 in both cancer subtype, whereas 1909 genes showed significant negative correlations (Fig. 5B).

Next, to uncover mechanisms regulated by SLC17A9, GO and KEGG pathway analysis of the co-expressed genes between LUAD and LUSC were performed using GSEA. GO term annotation showed that genes coexpressed with SLC17A9 were primarily involved in biological regulation, metabolic process and protein binding (Fig. 5C). KEGG analysis revealed enrichment in the metabolic process like glycosaminoglycan biosynthesis, linoleic acid metabolism, other glycan degradation, while processes, such as RNA transport, mismatch repair, and basal transcription factors, were inhibited (Fig. 5D). Furthermore, GSEA was conducted using oncogenic signatures and hallmark gene sets between SLC17A9 high and low expression in 46 NSCLC patients (GSE18842), respectively. As shown in Fig. $5 \mathrm{E}-\mathrm{H}$, the top significant hallmark gene sets including myc targets, DNA repair, coagulation and complement were enriched in high SLC17A9 expression group.

\section{Potential role of SLC17A9 -related hub genes and their prognostic value}

To identify the hub genes and understand the relationship between SLC17A9 expression and its potential mechanism in NSCLC, a PPI network consisting of 25 nodes was constructed based on the STRING database (Fig. 6A). The top 10 hub genes, namely SLC17A9, P2RX1, P2RX3, P2RX7, P2RX4, PANX1, WDTC1, ENTPD2, TRIM33 and PANX2, ranked by degrees of the CytoHubba plugin were selected (Fig. 6B). To further confirm whether the above hub genes were related to LUAD and LUSC, we analyzed the mRNA expression and prognostic values of the potential hub genes. The analysis demonstrated that these hub genes were significantly altered in LUAD and LUSC patients except for TRIM33 (Fig. 6C). While the P2RX1 was associated with the OS of LUAD patients, whereas the high expression of P2X4 and WDTC1 was associated with worse OS in LUSC patients (Fig. 6D).

\section{Knockdown of SLC17A9 inhibited cell proliferation of lung cancer cells in vitro}

To further verify the oncogenic role of SLC17A9 in lung cancer, we examined the effects of SLC17A9 knockdown on the human LUAD cell line A549. The efficiency of SLC17A9 siRNA mediated knockdown was confirmed via qRT-PCR and western blotting (Fig.7A). MTS and EdU assays showed that the cell proliferation rate of A549 cells was inhibited significantly after SLC17A9 knockdown (Fig. 7B-D). We also measured the intracellular ATP concentration. A decrease in ATP concentration was observed in the SLC17A9 knockdown group (Fig. 8C). In the cell cycle analyses, knockdown of SLC17A9 induced G1 arrest and decreased the numbers of A549 cells in the S phase (Fig. 7E). The real-time PCR results showed that SLC17A9 knockdown significantly increased the mRNA expression of MYC and cytochrome $C$, but decreased the mRNA expression of the P2X1 receptor, MFN2, and STAT3 compared to control (Fig. 7F). These finding may suggest that 
knockdown of SLC17A9 inhibited cell proliferation by up-regulation of MYC and cytochrome C and upregulation of P2X1 receptor, MFN2, and STAT3 in lung cancer cells.

\section{Upregulation of SLC17A9 involved in immune infiltration of patients with LUAD and LUSC}

Our GSEA analysis and previous study showed that high expression of SLC17A9 was involved in immune response [23]. Therefore, we investigated whether SLC17A9 expression correlated with immune infiltration in LUAD and LUSC using public databases. The 513 LUAD and 501 LUSC patients were divided into high and low groups based on the median value of SLC17A9 expression. The xCell correlation heatmap confirmed that SLC17A9 expression is significantly correlated with most of the CD8+ and CD4 ${ }^{+} T$ cells, plasma B cell, memory B cell, B cells, M1 macrophages and microenvironment score in LUAD (Fig. 8A). Our GSVA analysis revealed that NK cells, DC cells, Th17, Th1, and Th2 cells were significantly correlated with SLC17A9 expression (Fig. 8B). Specifically, SLC17A9 expression was associated with all most all the immune infiltrating cells in LUSC (Fig. 9A, B). Consistent with the xCell analysis results, it is remarkable that the expression of SLC17A9 was negatively correlated with tumor purity (cor $=-0.142, p<0.05$ ) and $C D 8^{+} T$ cells (cor $=-0.132, p<0.05)$, but positively correlated with $B$ cells (cor $=0.152, p<0.05)$ and $C D 4^{+} T$ cells $($ cor $=0.216$, $\mathrm{p}<0.05$ ), demonstrated using the TIMER web server in LUSC (Fig. 8C). Moreover, SLC17A9 expression was also negatively correlated with tumor purity $(\mathrm{cor}=-0.353, p<0.05)$ in LUSC, while it was positively correlated with B cells (cor $=0.262, p<0.05), C D 4^{+} T$ cells $(\operatorname{cor}=0.464, p<0.05)$, macrophages $($ cor $=0.203, p<0.05)$, neutrophil (cor $=0.257, p<0.05)$ and dentritic cells (cor $=0.327, p<0.05)$ (Fig. $9 \mathrm{C})$. These results indicated that SLC17A9 plays a specific role in immune infiltration in both LUAD and LUSC, especially for T cells and B cells.

\section{Correlation analysis between SLC17A9 expression and immune marker sets of patients with LUAD and LUSC}

To further investigate the relationship between SLC17A9 and the immune marker sets of various immune infiltrating cells, we first selected eight common checkpoint-related genes and examined the correlation of SLC17A9 with these genes using TCGA-LUAD and LUSC datasets. As is shown in Fig. 8D and Fig. 9D, the expression of CTLA4, LAG3, PDCD1 (also known as PD-1) and TIGIT was significantly elevated along with high SLC17A9 expression in patients with LUAD and LUSC $(p<0.05)$. The same was true for HAVCR2, PDCD1LG2, and SIGLEC15 in LUSC ( $p<0.05)$. We next focused on the correlations between SLC17A9 and gene marker sets of different types of immune cells in LUAD and LUSC in the TIMER database. The results revealed that purity adjustment had little effect on these association. Interestingly, we found that the SLC17A9 expression was significantly correlated with most innate and adaptive immune marker sets in LUSC with or with the correlation adjustment by purity (Table 4 and Table 5). However, the SLC17A9 expression level was significantly correlated with only 13 gene markers of innate immunity cells, including CD14 of monocytes, CD68 of tumor-associated macrophages, INOS and TNF-a of M1 macrophages, KIR2DL1, KIR3DL1, KIR3DL3 and KIR2DS4 of Natural killer cells, as well as CD11c and HLA-DQB1 of dendritic cells (Table 4). Marker sets of adaptive immunity cells, including general T cell markers (CD3D and CD3E), B cell markers (CD19, CD20 and CD23), Th1 markers (T-bet and TNF-a), Th2 markers (STAT6, STAT5A and IL13), Tfh marker (CD278), Th17 marker (IL17A), Treg (FOXP3, CCR8, STAT5B and TGF $\beta$ ), as well as PD-1, CTLA4 and LAG3 of T cell exhaustion have strong correlations with SLC17A9 expression in LUAD (Table 5).Taken 
together, these results showed that SLC17A9 was significantly associated with the expression of immunerelated genes in LUSC and LUAD.

Table 4. Correlation analysis between SLC17A9 and relate genes and markers of innate immunity cells in TIMER. 


\begin{tabular}{|c|c|c|c|c|c|c|c|c|c|}
\hline \multirow[t]{3}{*}{ Description } & \multirow[t]{3}{*}{ Gene markers } & \multicolumn{4}{|l|}{ LUAD } & \multicolumn{4}{|l|}{ LUSC } \\
\hline & & \multicolumn{2}{|l|}{ Purity } & \multicolumn{2}{|l|}{ None } & \multicolumn{2}{|l|}{ Purity } & \multicolumn{2}{|l|}{ None } \\
\hline & & Cor & $p$ & Cor & $p$ & Cor & $p$ & Cor & $p$ \\
\hline \multirow[t]{3}{*}{ Monocyte } & CD14 & 0.155 & * & 0.145 & * & 0.377 & * & 0.380 & * \\
\hline & CD86 & 0.082 & 0.068 & 0.066 & 0.133 & 0.322 & * & 0.324 & * \\
\hline & CD16(FCGR3A) & 0.050 & 0.269 & 0.045 & 0.311 & 0.300 & * & 0.308 & * \\
\hline \multirow[t]{3}{*}{ TAM } & CD68 & 0.112 & * & 0.106 & * & 0.241 & * & 0.251 & * \\
\hline & CCL2 & 0.075 & 0.096 & 0.075 & 0.088 & 0.303 & * & 0.307 & * \\
\hline & CCL5 & 0.022 & 0.619 & 0.022 & 0.617 & 0.306 & * & 0.318 & * \\
\hline \multirow{3}{*}{$\begin{array}{l}\text { M1 } \\
\text { Macrophage }\end{array}$} & INOS (NOS2) & 0.145 & * & 0.130 & * & 0.062 & 0.175 & 0.054 & 0.226 \\
\hline & CXCL10 & -0.013 & 0.770 & -0.015 & 0.741 & 0.168 & * & 0.179 & * \\
\hline & TNF-a (TNF) & 0.153 & * & 0.134 & * & 0.356 & * & 0.355 & * \\
\hline \multirow{3}{*}{$\begin{array}{l}\text { M2 } \\
\text { Macrophage }\end{array}$} & CD206(MRC1) & 0.014 & 0.750 & -0.011 & 0.798 & 0.343 & * & 0.331 & * \\
\hline & CD163 & 0.082 & 0.069 & 0.068 & 0.122 & 0.346 & * & 0.347 & * \\
\hline & IL10 & 0.069 & 0.126 & 0.054 & 0.218 & 0.319 & * & 0.325 & * \\
\hline \multirow[t]{4}{*}{ Neutrophils } & $\begin{array}{l}\text { CD66b } \\
\text { (CEACAM8) }\end{array}$ & -0.085 & 0.059 & -0.109 & * & 0.064 & 0.163 & 0.075 & 0.095 \\
\hline & $\begin{array}{l}\text { CD11b } \\
\text { (ITGAM) }\end{array}$ & 0.133 & * & 0.115 & * & 0.430 & * & 0.424 & * \\
\hline & CCR7 & 0.143 & * & 0.125 & * & 0.404 & * & 0.406 & * \\
\hline & CD15(FUT4) & 0.149 & * & 0.164 & * & 0.324 & * & 0.332 & * \\
\hline \multirow{7}{*}{$\begin{array}{l}\text { Natural killer } \\
\text { cell }\end{array}$} & KIR2DL1 & 0.109 & * & 0.104 & * & 0.077 & 0.095 & 0.103 & * \\
\hline & KIR2DL3 & 0.068 & 0.131 & 0.059 & 0.182 & 0.082 & 0.073 & 0.089 & * \\
\hline & KIR2DL4 & 0.056 & 0.215 & 0.062 & 0.161 & 0.085 & 0.063 & 0.106 & * \\
\hline & KIR3DL1 & 0.108 & * & 0.093 & * & 0.171 & * & 0.183 & * \\
\hline & KIR3DL2 & 0.048 & 0.290 & 0.048 & 0.274 & 0.099 & * & 0.113 & * \\
\hline & KIR3DL3 & 0.127 & * & 0.115 & * & 0.021 & 0.640 & 0.045 & 0.320 \\
\hline & KIR2DS4 & 0.093 & * & 0.080 & 0.071 & 0.143 & * & 0.155 & * \\
\hline $\begin{array}{l}\text { Dendritic } \\
\text { cell }\end{array}$ & HLA-DPB1 & 0.038 & 0.399 & 0.029 & 0.512 & 0.355 & * & 0.360 & * \\
\hline
\end{tabular}




\begin{tabular}{|lllllllll|}
\hline HLA-DQB1 & 0.100 & $*$ & 0.086 & 0.052 & 0.302 & $*$ & 0.302 & $*$ \\
\hline HLA-DRA & -0.028 & 0.531 & -0.041 & 0.356 & 0.307 & $*$ & 0.314 & $*$ \\
\hline HLA-DPA1 & 0.000 & 0.998 & -0.009 & 0.845 & 0.337 & $*$ & 0.341 & $*$ \\
\hline BDCA-1(CD1C) & 0.040 & 0.373 & 0.022 & 0.616 & 0.197 & $*$ & 0.193 & $*$ \\
\hline BDCA-4(NRP1) & -0.028 & 0.531 & -0.038 & 0.387 & 0.325 & $*$ & 0.328 & $*$ \\
\hline CD11c (ITGAX) & 0.314 & $*$ & 0.303 & $*$ & 0.518 & $*$ & 0.517 & $*$ \\
\hline NKp46(NCR1) & 0.070 & 0.119 & 0.068 & 0.124 & 0.257 & $*$ & 0.263 & $*$ \\
\hline
\end{tabular}

TAM, tumorassociated macrophage; Cor, R value of Spearman's correlation; None, correlation without adjustment. Purity, correlation adjusted by purity. ${ }^{*} \mathrm{P}<0.05$.

Table 5. Correlation analysis between CAPRIN1 and relate genes and markers of adaptive immunity cells in TIMER. 


\begin{tabular}{|c|c|c|c|c|c|c|c|c|c|}
\hline \multirow[t]{3}{*}{ Description } & \multirow[t]{3}{*}{ Gene markers } & \multicolumn{4}{|l|}{ LUAD } & \multicolumn{4}{|l|}{ LUSC } \\
\hline & & \multicolumn{2}{|l|}{ Purity } & \multicolumn{2}{|l|}{ None } & \multicolumn{2}{|l|}{ Purity } & \multicolumn{2}{|l|}{ None } \\
\hline & & Cor & $p$ & Cor & $p$ & Cor & $p$ & Cor & $p$ \\
\hline \multirow{2}{*}{$\mathrm{CD} 8+\mathrm{T}$ cell } & CD8A & 0.031 & 0.489 & 0.028 & 0.527 & 0.253 & * & 0.263 & * \\
\hline & CD8B & 0.063 & 0.159 & 0.057 & 0.193 & 0.228 & * & 0.234 & * \\
\hline \multirow{3}{*}{$\begin{array}{l}\text { T cell } \\
\text { (general) }\end{array}$} & CD3D & 0.098 & * & 0.089 & * & 0.319 & * & 0.332 & * \\
\hline & CD3E & 0.101 & * & 0.096 & * & 0.362 & * & 0.369 & * \\
\hline & CD2 & 0.086 & 0.057 & 0.076 & 0.087 & 0.341 & * & 0.350 & * \\
\hline \multirow[t]{4}{*}{ B cell } & CD19 & 0.246 & * & 0.243 & * & 0.445 & * & 0.450 & * \\
\hline & CD20(MS4A1) & 0.171 & * & 0.162 & * & 0.332 & * & 0.337 & * \\
\hline & CD138(SDC1) & 0.046 & 0.309 & 0.049 & 0.263 & -0.146 & * & -0.152 & * \\
\hline & CD23(FCER2) & 0.169 & * & 0.155 & * & 0.326 & * & 0.323 & * \\
\hline \multirow[t]{5}{*}{ Th1 } & T-bet (TBX21) & 0.171 & * & 0.164 & * & 0.400 & * & 0.411 & * \\
\hline & STAT4 & 0.070 & 0.122 & 0.069 & 0.117 & 0.411 & * & 0.420 & * \\
\hline & STAT1 & -0.002 & 0.969 & -0.003 & 0.953 & 0.176 & * & 0.194 & * \\
\hline & IFN-Y (IFNG) & 0.056 & 0.217 & 0.045 & 0.313 & 0.150 & * & 0.165 & * \\
\hline & TNF-a (TNF) & 0.153 & * & 0.134 & * & 0.356 & * & 0.355 & * \\
\hline \multirow[t]{4}{*}{ Th2 } & GATA3 & 0.061 & 0.177 & 0.059 & 0.182 & 0.349 & * & 0.345 & * \\
\hline & STAT6 & 0.158 & * & 0.149 & * & 0.158 & * & 0.162 & * \\
\hline & STAT5A & 0.226 & * & 0.216 & * & 0.432 & * & 0.440 & * \\
\hline & IL13 & 0.122 & * & 0.107 & * & 0.119 & * & 0.124 & * \\
\hline \multirow[t]{4}{*}{ Tfh } & BCL6 & 0.081 & 0.072 & 0.082 & 0.064 & 0.019 & 0.681 & 0.005 & 0.906 \\
\hline & IL21 & 0.060 & 0.183 & 0.042 & 0.346 & 0.195 & * & 0.194 & * \\
\hline & CD278(ICOS) & 0.093 & * & 0.080 & 0.069 & 0.362 & * & 0.370 & * \\
\hline & CXCL13 & 0.085 & 0.060 & 0.090 & * & 0.320 & * & 0.316 & * \\
\hline \multirow[t]{2}{*}{ Th17 } & STAT3 & 0.022 & 0.627 & 0.020 & 0.647 & 0.173 & * & 0.174 & * \\
\hline & IL17A & 0.131 & * & 0.124 & * & 0.043 & 0.348 & 0.048 & 0.288 \\
\hline \multirow[t]{3}{*}{ Treg } & FOXP3 & 0.182 & * & 0.173 & * & 0.426 & * & 0.430 & * \\
\hline & CCR8 & 0.091 & * & 0.076 & 0.085 & 0.353 & * & 0.358 & * \\
\hline & STAT5B & 0.114 & * & 0.102 & * & 0.195 & * & 0.200 & * \\
\hline
\end{tabular}




\begin{tabular}{|c|c|c|c|c|c|c|c|c|c|}
\hline & TGF $\beta$ (TGFB1) & 0.181 & * & 0.185 & * & 0.293 & * & 0.288 & * \\
\hline & CD25(IL2RA) & 0.047 & 0.292 & 0.038 & 0.390 & 0.313 & * & 0.324 & * \\
\hline \multirow{5}{*}{$\begin{array}{l}\text { T cell } \\
\text { exhaustion }\end{array}$} & PD-1 (PDCD1) & 0.231 & * & 0.229 & * & 0.420 & * & 0.430 & * \\
\hline & CTLA4 & 0.183 & * & 0.171 & * & 0.405 & * & 0.413 & * \\
\hline & LAG3 & 0.172 & * & 0.170 & * & 0.309 & * & 0.320 & * \\
\hline & $\begin{array}{l}\text { TIM-3 } \\
\text { (HAVCR2) }\end{array}$ & 0.086 & 0.057 & 0.069 & 0.117 & 0.314 & * & 0.322 & * \\
\hline & GZMB & 0.088 & 0.050 & 0.088 & * & 0.250 & * & 0.262 & * \\
\hline
\end{tabular}

Cor, R value of Spearman's correlation; None, correlation without adjustment. Purity, correlation adjusted by purity. ${ }^{*} \mathrm{P}<0.05$.

\section{Discussion}

The involvement of the SLC17 family of membrane transporters has been reported in various cancers, including colorectal cancer [12], gastric carcinoma [24], and acute myeloid leukemia [25]. To date, few studies have evaluated the expression pattern and prognosis value of SLC17As in NSCLC. In the present study, we comprehensively depicted the expression pattern, mutation, prognostic values, and diagnostic values of SLC17As using data from thousands of LUAD and LUSC samples from TCGA. It revealed that most SLC17As were slightly downregulated in human LUAD and LUSC and may be used as diagnostic biomarkers for distinguishing LUAD or LUSC patients from healthy individuals based on ROC analysis. Additionally, we combined further bioinformatics and in vitro analyses to investigate the oncogenic function of SLC17A9. Collectively, we highlighted that SLC17A9 can be used as a prognostic marker and a potential therapeutic target for LUAD and LUSC.

It has been reported that the mRNA expression of SLC17A5 increased in a cisplatin-resistant gastric cancer cell line [26]. SLC17A5 expression was obviously lower in tumor tissues compared with normal tissues in our study. However, we did not find a significant correlation between SLC17A5 expression levels and prognosis in human LUAD and LUSC. Therefore, whether SLC17A5 could serve as a potential diagnostic and prognostic marker deserves further clinical validation. Genetic mutations (such as missense and truncating mutations) in SLC17As occur frequently and usually cause phenotypic changes, which were associated with human NSCLC $[13,27]$. Previous studies have described and identified several disease-causing mutations in SLC17As (http://www.bioparadigms.org.). Mutation analysis has demonstrated that the mutations in SLC17A1 and SLC17A3 were linked to gout. The mutations in SLC17A5 mainly caused severe neurologic symptoms [11], and mutations at the DFNA25 locus in SLC17A8 (VGLUT3) have been detected in patients with nonsyndromic deafness [28]. SLC17A9 (G78R) was detected as novel somatic gene mutation in early NSCLC [29]. However, no other mutation in SLC17A2/3/4/8 was reported. In the present study, the mutation frequency of all SLC17As (ranged 1.1-4.0\%) was reported in LUAD and LUSC patients. Furthermore, the study identified missense mutations with the highest functional impact scores mainly in SLC17A1/4/6/8. 
Increased expression of SLC17A9 was reported to be associated with poorer survival in patients gastrointestinal cancer including gastric [24], liver [30], colorectal cancers [12]. Consistent with these findings, we observed that only the mRNA levels of SLC17A9 were significantly higher in human LUAD and LUSC, compared to adjacent normal tissues in TCGA and GEO databases. However, SLC17A9 expression did not show any prognostic relevance to clinicopathological parameters. Additionally, lower expression of SLC17A9 was associated with worse prognosis for LUAD patients in TCGA database. However, SLC17A9 upregulation was demonstrated as an unfavorable prognostic factor for patients with LUSC. The immune function and genetic mutation frequency of SLC17A9 may explain some discrepancies between our analysis and empirical results.

To investigate the oncogenic mechanism of SLC17A9, we analyzed the SLC17A9 co-expression network in LUAD and LUSC using TCGA data. Our results suggested that the functions of SLC17A9 mainly included biological regulation, metabolic process (such as glycosaminoglycan biosynthesis and linoleic acid metabolism), and protein binding, while inhibiting RNA transport and mismatch repair. These results were further validated via GSEA, which revealed that oncogenic signaling pathways and hallmark of myc targets, DNA repair, coagulation, and complement were related to SLC17A9 upregulation in 46 NSCLC patients. All these pathways were implicated in cancer cell growth and proliferation [31, 32]. SLC17A9 mRNA expression reportedly correlated positively with a TP53 mutation that could modulate DNA damage response, RNA transport, and protein binding [33]. SLC17A9 silencing reportedly inhibited the viability of C2C12, COS1, and HEK293T cells. Takai et al. [34] showed that TGF- $\beta 1$ failed to induce release of ATP from SLC17A9knockdown cells. Here, we demonstrated that SLC17A9 knockdown inhibited cell proliferation in A549 cells by affecting MYC, cytochrome C, MFN2, and STAT3, and it also decreased the production of ATP by the P2X1 receptor. Although the mechanism underlying SLC17A9 regulation requires further investigation, the previous and present data strongly suggested that SLC17A9 potentially promotes the progression of LUAD and LUSC through its effects on the cell cycle, cell proliferation, and DNA damage.

Recent studies have revealed that SLC17A9 acted as an essential component for vesicular ATP release [35]. ATP could interact with the ionotropic P2X1-7 receptors and the metabotropic P2Y1-14 receptors [36]. Zhong et al. provided a regulatory mechanism, which suggested that SLC17A9 could transport ATP into the lysosome and activate liposomal P2X4 [37]. Consistent with previous study, hub genes such as P2RX1, P2RX3, P2RX7 and P2RX4, which were receptors for ATP that acted as a ligand-gated ion channel, were identified with a higher node degree in the PPI network. In recent studies, ATP receptors have also been closely associated with diseases in central nervous system [38] and different tumors [39, 40]. Chong et al. [41] demonstrated that mRNA expression of P2X receptor members including P2X1, P2X4, P2X5 and P2X7 increased in Chinese pediatric acute leukemia patients versus controls. P2X7 receptor expression has been reported to be predictor for lymph node metastasis in thyroid carcinoma. In fact, P2X7 activation potentially augmented breast and LUAD-derived A549 invasiveness [42]. Thus, we also analyzed the expression of the hub genes and explored their potential prognostic values in LUAD and LUSC. Results from our study showed that mRNA expression of all the hub genes were significantly changed. ATP receptors such as P2RX1, P2RX7 and P2RX4 were significantly down-regulated in LUAD and LUSC. Further, dysregulation of P2RX1 and WDTC1 was correlated with poor prognosis in patients with LUAD. The mRNA levels of P2RX4 and WDTC1 was significantly lower and related to poor survival for patients with LUSC.

Page $19 / 32$ 
In general, the NSCLC TME played a key role in tumor progression and metastasis [43]. Previous study has demonstrated that SLC17A9 involved in T cell receptor (TCR)-dependent ATP release [44]. Our GSEA analysis further reported that HALLMARK_COMPLEMETN of immune-related pathway was enriched in SLC17A9 high group. In this study, we investigated the relationship between SLC17A9 expression and immune cell infiltration levels in the TCGA-LUAD and LUSC tumor samples. We found that SLC17A9 just showed strong association with most gene markers and a large population of immune cells in LUSC, including immune checkpoint-related genes, supporting the observation that overall survival of SLC17A9 lower expression group was significantly worse verse to high expression group. In addition, SLC17A9 was closely related to partial B cells and $C D 8^{+}$T cells and not with neutrophils in LUAD. Additionally, our results showed that SLC17A9 is related to innate immunity and adaptive immunity in LUAD. Therefore, SLC17A9 may improve lung cancer patient prognosis through immune infltration and activation, especially in LUSC. However, the role of SLC17A9 in the infiltration and activation of NK cells,macrophages and T cell in lung cancer is still controversial $\square$ and the exact role of SLC17A9 in TME of LUAD and LUSC remains further investigation.

In conclusions, SLC17A9 and the hub genes identified herein play key roles in cancer progression, and were related to poor prognosis for patients with LUAD and LUSC. Moreover, biological regulation, immune infiltration, metabolic process, myc targets and DNA repair may enriched in NSCLC regulated by SLC17A9. In vitro analyses revealed that SLC17A9 might promote LUAD progression proliferation through upregulating the expression of MYC and cytochrome $\mathrm{C}$. The limitation lies in the fact that bioinformatic analysis is a powerful tool to highlight molecular mechanisms underlying SLC17A9, protein expression analysis and experimental validations is warranted to further validate our findings and explore the detailed mechanism at molecular and cellular levels. Together, these results suggest that SLC17A9 contributes to the progression of LUAD and LUSC, and can be used as a novel prognostic marker and therapy target for immunotherapy.

\section{Declarations}

\section{Ethics approval and consent to participate}

Not applicable.

\section{Consent for publication}

Not applicable.

\section{Availability of data and materials}

Publicly available datasets were analyzed in this study. The datasets generated for this study could be found in TCGA (https://portal.gdc.cancer.gov/projects/TCGA-LUAD and LUSC) and GEO (https://www.ncbi.nlm.nih.gov/geo). The materials and other data not included in the article are available from the corresponding author on reasonable request.

\section{Competing interests}

The authors declare that they have no competing interests. 


\section{Funding}

This work was supported by the Hubei province's Outstanding Medical Academic Leader program, the Foundation for Innovative Research Team of Hubei Provincial Department of Education (No. T2020025), the Hubei Provincial Department of Science and Technology Innovation Group Program (No. 2019CFA034), Innovative Research Program for Graduates of Hubei University of Medicine (No. YC2020011) and the Key Discipline Project of Hubei University of Medicine.

\section{Authors' contributions}

Yan Gao, Changbin Ke and Zhijun Pei conceived and designed the study. Zhijun Pei, Yijia Chen Fuyan Li and Rumin Wu acquired and managed funding. Yan Gao, Daobing Zeng, Fan Tan, Xusheng Liu, Xiaohui Li and Yang Li acquired, collected, and extracted the data included in this analysis. Yan Gao, Yijia Chen, Shanchun Su, Jianwei Yang, Lumeng Zhou analyzed data. Yan Gao and Yijia Chen originally drafted the paper, all authors helped with review \&editing.

Acknowledgments

We thanked Juan Xue (Hubei University of Medicine) for her kindly help in flow cytometry analysis.

\section{References}

1. Sung H, Ferlay J, Siegel RL, Laversanne M, Soerjomataram I, Jemal A, Bray F: Global cancer statistics 2020: GLOBOCAN estimates of incidence and mortality worldwide for 36 cancers in 185 countries. $C A$ : $A$ Cancer Journal for Clinicians 2021.

2. Herbst RS, Morgensztern $D$, Boshoff $C$ : The biology and management of non-small cell lung cancer. Nature (London) 2018, 553(7689):446-454.

3. Zhou L, Yuan L, Gao Y, Liu X, Dai Q, Yang J, Pei Z: Nucleophosmin 1 overexpression correlates with 18FFDG PET/CT metabolic parameters and improves diagnostic accuracy in patients with lung adenocarcinoma. EUR J NUCL MED MOL I 2020.

4. George J, Lim JS, Jang SJ, Cun Y, Ozretić L, Kong G, Leenders F, Lu X, Fernández-Cuesta L, Bosco G et al: Comprehensive genomic profiles of small cell lung cancer. NATURE 2015, 524(7563):47-53.

5. Akhavanfard S, Padmanabhan R, Yehia L, Cheng F, Eng C: Comprehensive germline genomic profiles of children, adolescents and young adults with solid tumors. NAT COMMUN2020, 11(1).

6. Neapolitan R, Horvath CM, Jiang X: Pan-cancer analysis of TCGA data reveals notable signaling pathways. BMC CANCER 2015, 15(1).

7. Jiang $\mathrm{H}, \mathrm{Xu} \mathrm{S}$, Chen $\mathrm{C}$ : A ten-gene signature-based risk assessment model predicts the prognosis of lung adenocarcinoma. BMC CANCER 2020, 20(1).

8. Byun DJ, Wolchok JD, Rosenberg LM, Girotra M: Cancer immunotherapy - immune checkpoint blockade and associated endocrinopathies. NAT REV ENDOCRINOL 2017, 13(4):195-207.

9. Sun R, Limkin EJ, Vakalopoulou M, Dercle L, Champiat S, Han SR, Verlingue L, Brandao D, Lancia A, Ammari $S$ et al: A radiomics approach to assess tumour-infiltrating CD8 cells and response to anti-PD-1 
or anti-PD-L1 immunotherapy: an imaging biomarker, retrospective multicohort study. The lancet oncology 2018, 19(9):1180-1191.

10. Hellmann MD, Nathanson T, Rizvi H, Creelan BC, Sanchez-Vega F, Ahuja A, Ni A, Novik JB, Mangarin LMB, Abu-Akeel $\mathrm{M}$ et al: Genomic Features of Response to Combination Immunotherapy in Patients with Advanced Non-Small-Cell Lung Cancer. CANCER CELL 2018, 33(5):843-852.

11. Reimer RJ: SLC17: A functionally diverse family of organic anion transporters. MOL ASPECTS MED 2013, 34(2-3):350-359.

12. Yang L, Chen Z, Xiong W, Ren H, Zhai E, Xu K, Yang H, Zhang Z, Ding L, He Y et al: High expression of SLC17A9 correlates with poor prognosis in colorectal cancer. HUM PATHOL 2019, 84:62-70.

13. Cao Q, Zhao K, Zhong XZ, Zou Y, Yu H, Huang P, Xu T, Dong X: SLC17A9 Protein Functions as a Lysosomal ATP Transporter and Regulates Cell Viability. J BIOL CHEM 2014, 289(33):23189-23199.

14. Mihara H, Uchida K, Koizumi S, Moriyama Y: Involvement of VNUT-exocytosis in transient receptor potential vanilloid 4-dependent ATP release from gastrointestinal epithelium. PLOS ONE 2018, 13(10):e206276.

15. Tokunaga A, Tsukimoto M, Harada H, Moriyama Y, Kojima S: Involvement of SLC17A9-dependent Vesicular Exocytosis in the Mechanism of ATP Release during T Cell Activation. J BIOL CHEM 2010, 285.

16. Klaus, Galensa: ggplot2: elegant graphics for data analysis (2nd ed.). Computing Reviews 2017.

17. Nagy D, Munkácsy G, Gyrffy B: Pancancer survival analysis of cancer hallmark genes. SCI REP-UK 2020.

18. Gao J, Aksoy BA, Dogrusoz U, Dresdner G, Gross B, Sumer So, Sun Y, Jacobsen A, Sinha R, Larsson E et al: Integrative Analysis of Complex Cancer Genomics and Clinical Profiles Using the cBioPortal. SCI SIGNAL 2013, 6(269):I1.

19. Vasaikar SV, Peter S, Jing W, Bing Z: LinkedOmics: analyzing multi-omics data within and across $\mathbf{3 2}$ cancer types. NUCLEIC ACIDS RES 2017(D1):D1.

20. Damian S, Andrea F, Michael K, Milan S, Alexander R, Pablo M, Tobias D, Manuel S, Jean M, Peer B: The STRING database in 2011: functional interaction networks of proteins, globally integrated and scored. NUCLEIC ACIDS RES 2011, 39(Database issue):561-568.

21. Li T, Fan J, Wang B, Traugh N, Chen Q, Liu JS, Li B, Liu XS: TIMER: A Web Server for Comprehensive Analysis of Tumor-Infiltrating Immune Cells. CANCER RES 2017, 77(21):e108.

22. Liu X, Huang H, Gao Y, Zhou L, Yang J, Li X, Li Y, Zhao H, Su S, Ke C et al: Visualization of gene therapy with a liver cancer-targeted adeno-associated virus 3 vector. J CANCER 2020, 11(8):2192-2200.

23. Tokunaga A, Tsukimoto M, Harada H, Moriyama Y, Kojima S: Involvement of SLC17A9-dependent Vesicular Exocytosis in the Mechanism of ATP Release during T Cell Activation. J BIOL CHEM 2010, 285(23):17406-17416.

24. Li J, Su T, Yang L, Deng L, Zhang C, He Y: High SLC17A9 expression correlates with poor survival in gastric carcinoma. FUTURE ONCOL 2019, 15(36):4155-4166.

25. Lee C, Park J, Oh JI, Kim B, Kwon JH, Kim BK, Ahn K, Yoon S: Abstract 3540: Down-regulation of solute carrier family 17 member 1(SLC17A1) expression contributes to chemotherapy resistance in acute myeloid leukemia. Cancer research (Chicago, III.) 2010, 70(8 Supplement):3540. 
26. Zhang $Y$, Wu $Q$, Xiao X, Li D, Wang X: Silencing MRP4 by small interfering RNA reverses acquired DDP resistance of gastric cancer cell. CANCER LETT 2010, 291(1):76-82.

27. Devarakonda S, Rotolo F, Tsao MS, Lanc I, Brambilla E, Masood A, Olaussen KA, Fulton R, Sakashita S, Mcleer-Florin A: Tumor Mutation Burden as a Biomarker in Resected Non-Small-Cell Lung Cancer. J CLIN ONCOL 2018, 36(30):02018781963.

28. Ruel J, Emery S, Nouvian R, Bersot T, Amilhon B, Rybroek JMV, Rebillard G, Lenoir M, Eybalin M, Delprat B: Impairment of SLC17A8 encoding vesicular glutamate transporter-3, VGLUT3, underlies nonsyndromic deafness DFNA25 and inner hair cell dysfunction in null mice. AM J HUM GENET 2008, 83(2):278-292.

29. Sakashita S, Masahiro M, Matsuoka R, Muratani M, Noguchi M: P1.09-16 Novel Somatic Gene Mutation of SLC17A9, Detected in Early-Stage Lung Adenocarcinoma. J THORAC ONCOL 2018, 13(10):S556.

30. Wu J, Yang Y, Song J: Expression of SLC17A9 in hepatocellular carcinoma and its clinical significance. ONCOL LETT 2020, 20(5):1.

31. Sanchez-Vega F, Mina M, Armenia J, Chatila WK, Mariamidze A: Oncogenic Signaling Pathways in The Cancer Genome Atlas. CELL 2018, 173(2):321-337.

32. Lord CJ, Ashworth A: The DNA damage reponse and cancer therapy. NATURE 2012, 481(7381):287-294.

33. Gupta S, Silveira DA, Mombach JCM: Towards DNA-damage induced autophagy: A Boolean model of p53-induced cell fate mechanisms. DNA REPAIR 2020, 96(December 2020):102971.

34. Takai E, Tsukimoto M, Harada H, Sawada K, Moriyama Y, Kojima S: Autocrine regulation of TGF- 1induced cell migration by exocytosis of ATP and activation of P2 receptors in human lung cancer cells. $J$ CELL SCI 2012, 125(21):5051-5060.

35. Hasuzawa N, Moriyama S, Moriyama Y, Nomura M: Physiopathological roles of vesicular nucleotide transporter (VNUT), an essential component for vesicular ATP release. Biochimica et Biophysica Acta (BBA) - Biomembranes 2020, 1862(12):183408.

36. Schenk U, Frascoli M, Proietti M, Geffers R, Traggiai E, Buer J, Ricordi C, Westendorf AM, Grassi F: ATP Inhibits the Generation and Function of Regulatory T Cells Through the Activation of Purinergic P2X Receptors. SCI SIGNAL 2011, 4(162):a12.

37. Zhong XZ, Cao Q, Sun X, Dong X: Activation of lysosomal P2X4 by ATP transported into lysosomes via VNUT/SLC17A9 using V-ATPase generated voltage gradient as the driving force. The Journal of Physiology 2016, 594(15):4253-4266.

38. Rivera A, Vanzulli I, Butt AM: A Central Role for ATP Signalling in Glial Interactions in the CNS. CURR DRUG TARGETS 2016, 17(16):1829.

39. Adinolfi E, Capece M, Amoroso F, Marchi ED, Franceschini A: Emerging Roles of P2X Receptors in Cancer. CURR MED CHEM 2015, 22(7):--

40. Virgilio FD, Vultaggio-Poma V, Sarti AC: P2X receptors in cancer growth and progression. BIOCHEM PHARMACOL 2020.

41. Gu L, Li F, Zhao L, Liu Y, Chu Q, Zang X, Liu J, Ning G, Zhao Y: Association of XIAP and P2X7 receptor expression with lymph node metastasis in papillary thyroid carcinoma. ENDOCRINE 2010, 38(2):276-282.

42. Adinolfi E, Capece M, Amoroso F, De Marchi E, Franceschini A: Emerging roles of P2X receptors in cancer. CURR MED CHEM 2015, 22(7):878. 
43. Sheng W, LaFleur MW, Nguyen TH, Chen S, Chakravarthy A, Conway JR, Li Y, Chen H, Yang H, Hsu PH et al: LSD1 Ablation Stimulates Anti-tumor Immunity and Enables Checkpoint Blockade. CELL 2018, 174(3):549-563.

44. Tokunaga A, Tsukimoto M, Harada H, Moriyama Y, Kojima S: Involvement of SLC17A9-dependent Vesicular Exocytosis in the Mechanism of ATP Release during T Cell Activation. J BIOL CHEM 2010, 285(23):17406-17416.

\section{Figures}
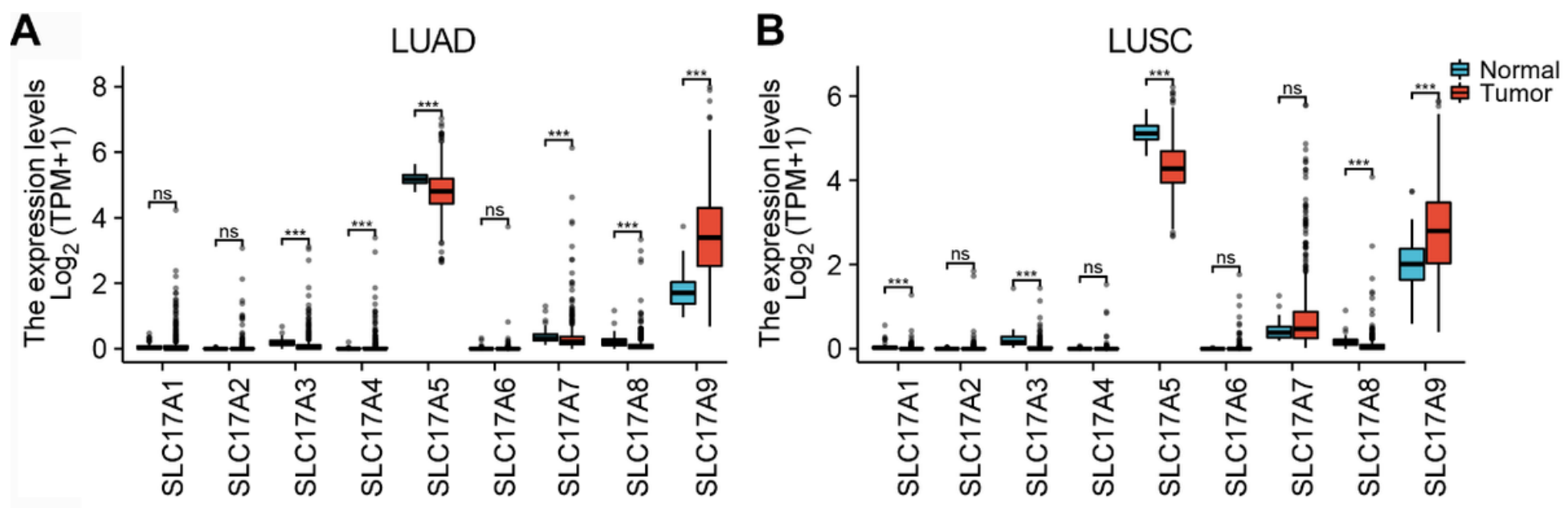

C

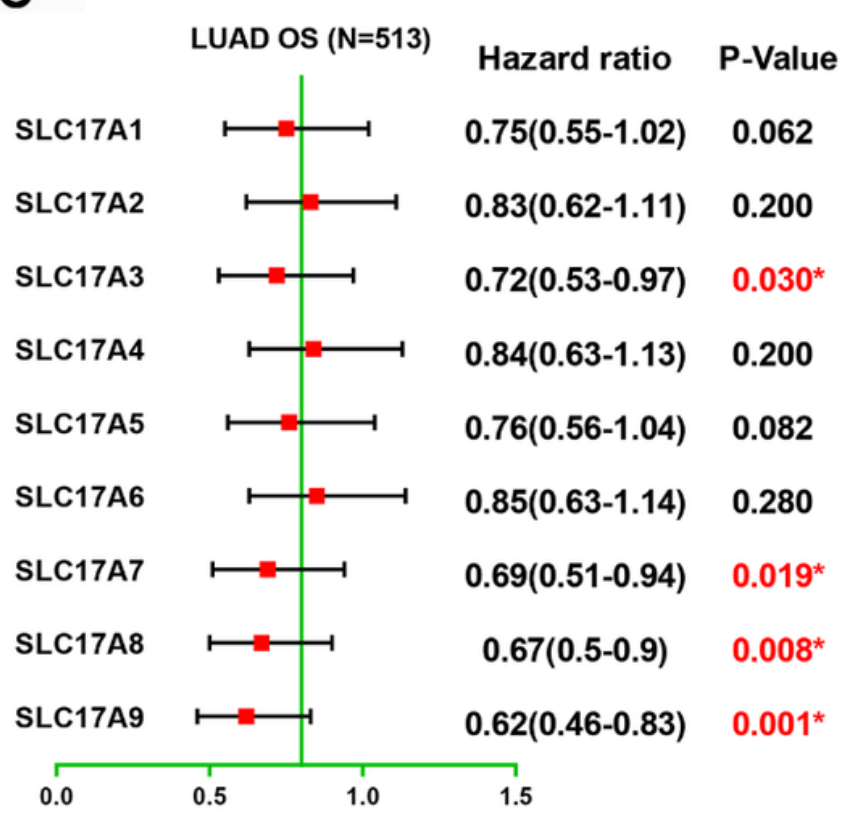

D

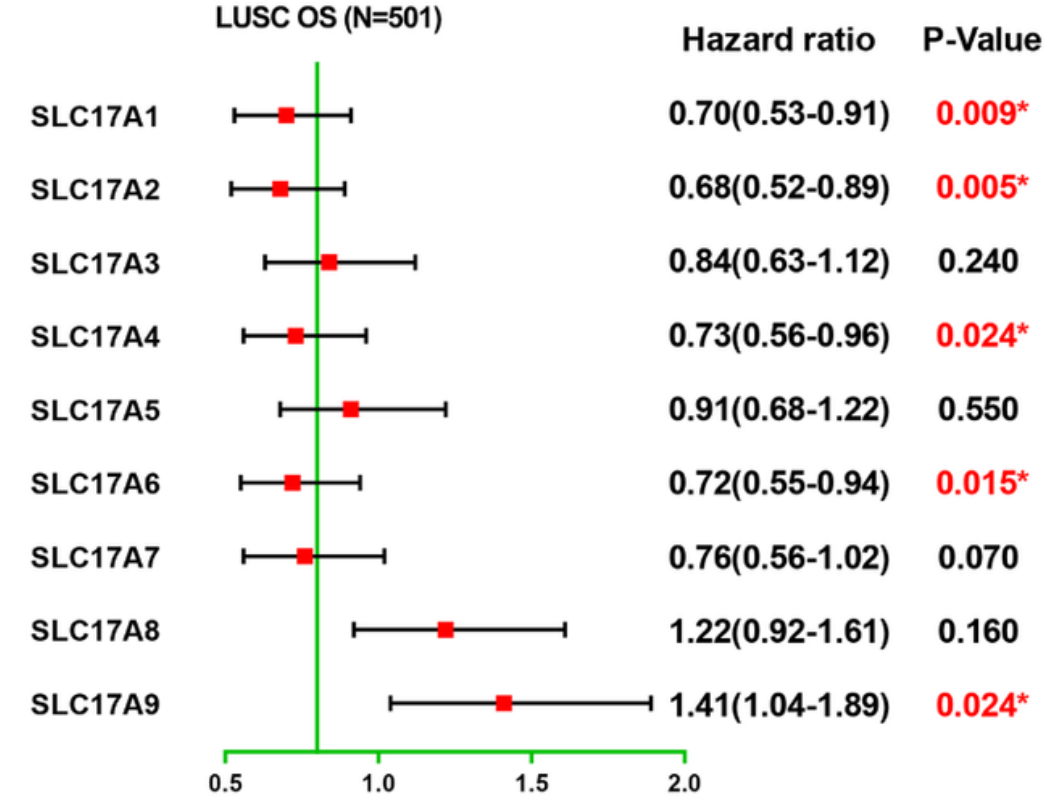

Figure 1

Expression and overall survival characteristics of SLC17As in human LUAD and LUSC tissues. (A) Relative mRNA levels of nine SLC17As across tumor and normal tissues in LUAD and LUSC (B) were analyzed using TCGA databases, The t-test was used to estimate the significance of difference in gene expression levels between groups (ns, ${ }^{*} p<0.05$; $\left.* \star p<0.01 ; * \star \star p<0.001\right)$. (C) Forest plot of hazard ratios depicting overall survival of SLC17A genes in LUAD and LUSC (D) (red values * $p<0.05)$. 

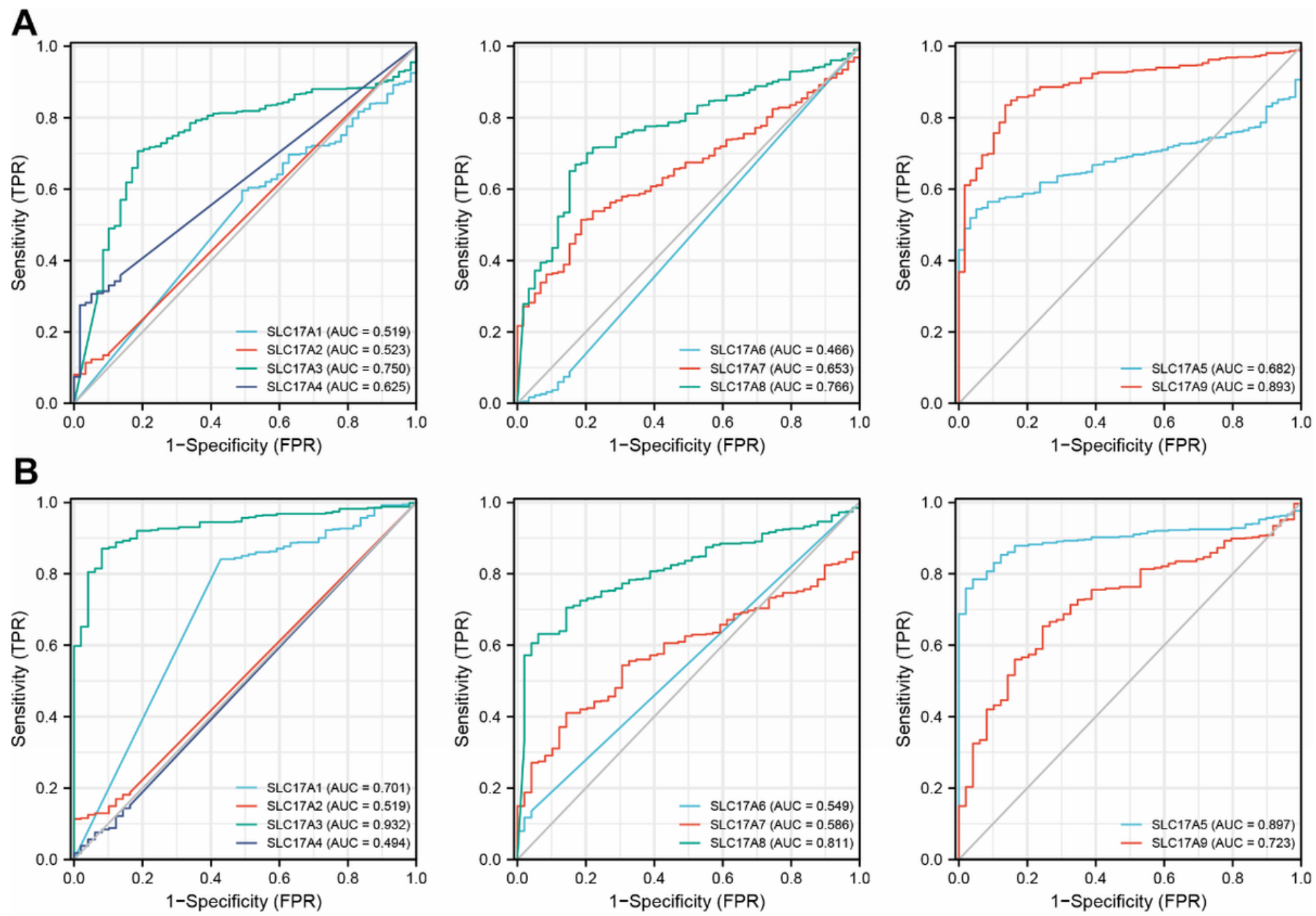

Figure 2

The ROC curve of individual SLC17A family genes including type I phosphate transporters, vesicular glutamate transporters, sialin and VNUT in patients with LUAD (A) and LUSC (B). 
A

Study of origin

SLC17A1 2.4\%

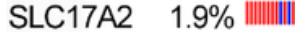

SLC17A3 $2.2 \%$ || ||

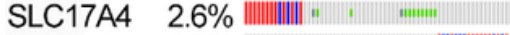

SLC17A5 $1.9 \%$

SLC17A6 2.4\%

SLC17A7 $1.1 \%$

SLC17A8 $2.2 \%$

SLC17A9 $4.0 \%$
Genetic Alteratic

Genetic Alteratic

Study of origin

B

SLC17A1 SLC17A2 SLC17A3

| Deep Deletion (unknown significance) | No alterations - Not profiled
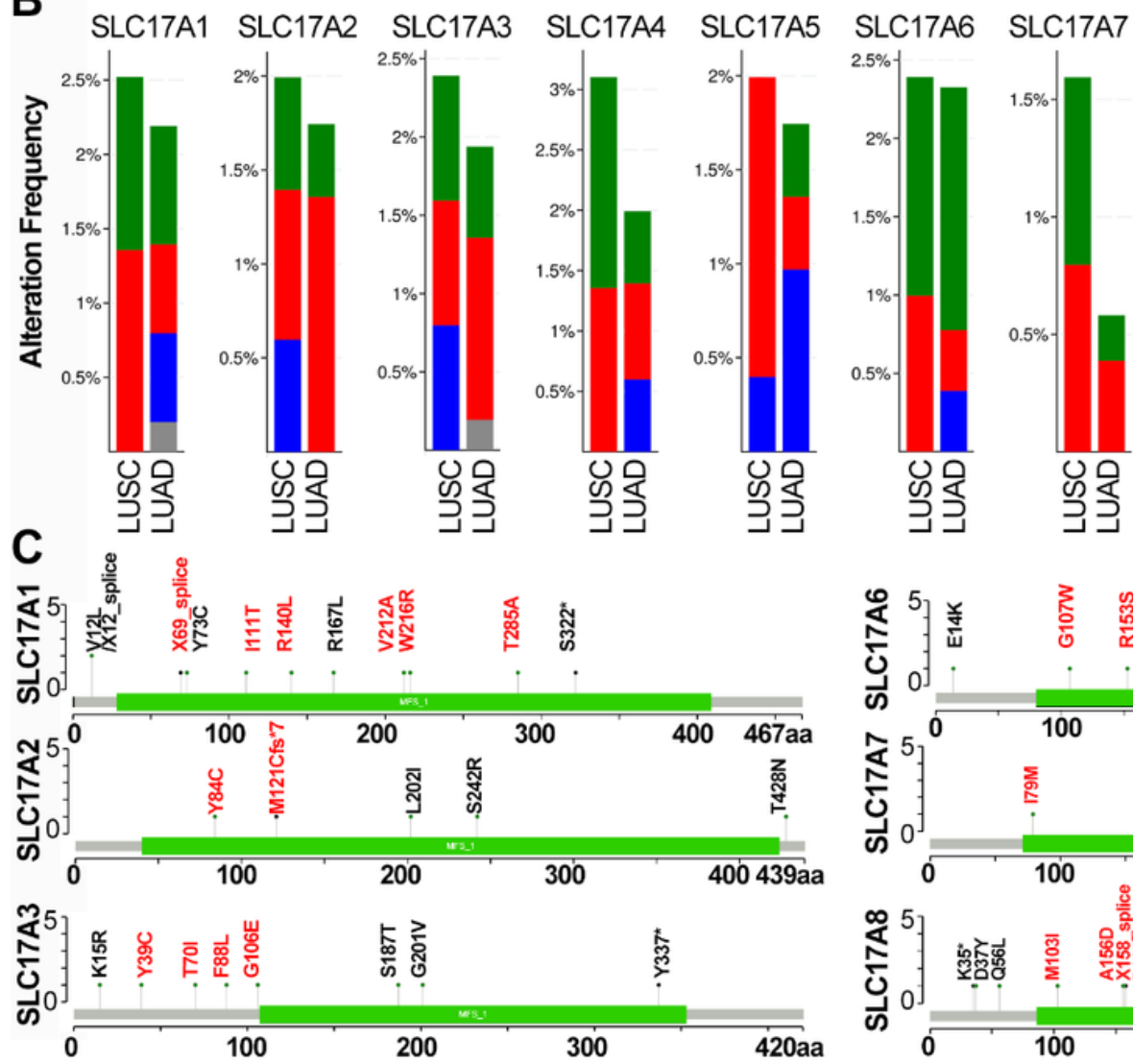

SLC17A8 SLC17A9
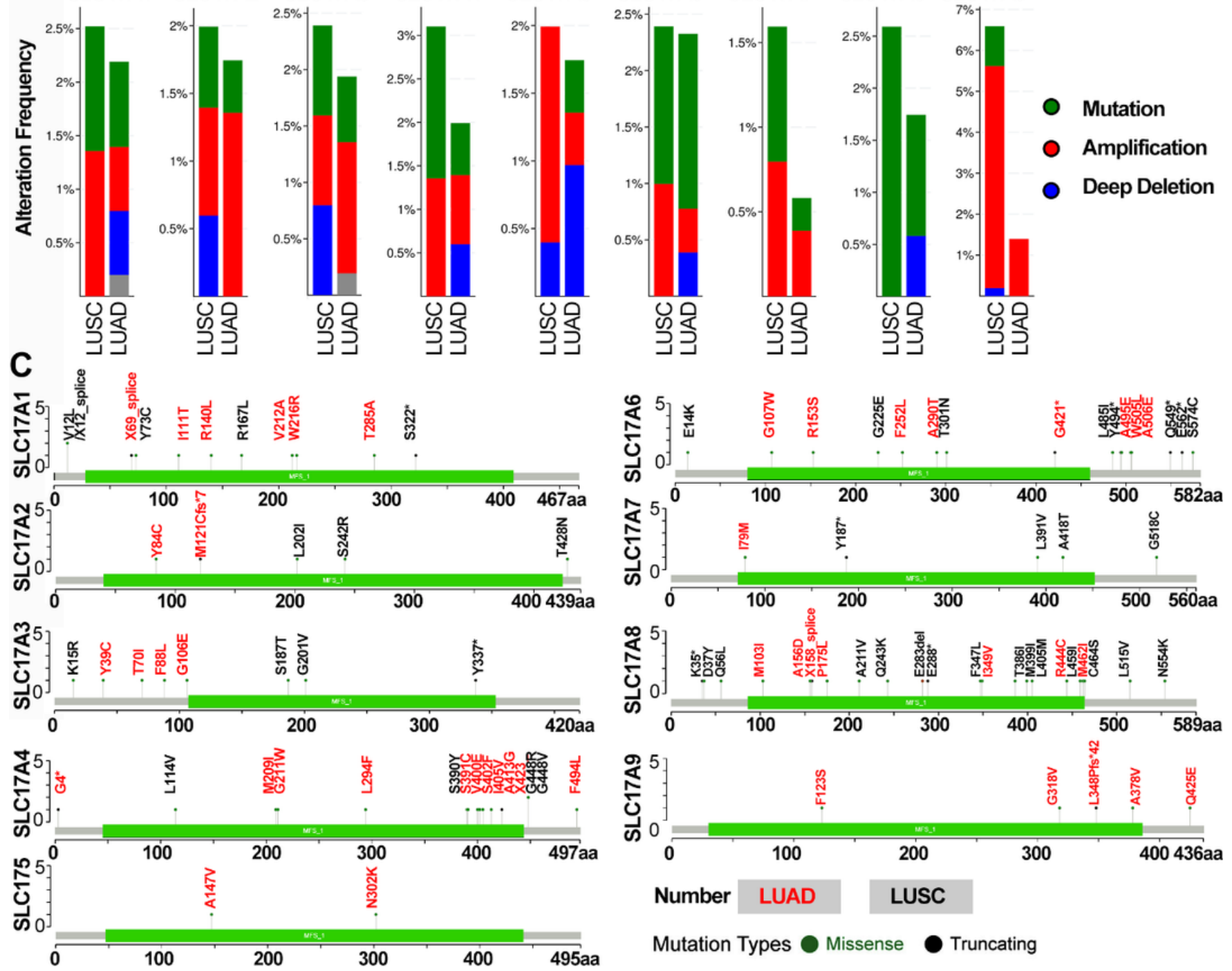

Figure 3

Summary of genetic mutations in SLC17A family genes in LUAD and LUSC by cBioPortal. (A) The total alteration of frequency were colored by the type in LUAD and LUSC studies. (B) The mutation profile and putative copy-number alterations (CNAs) represented as columns in TCGA cohort. (C) The structured domains and the location of fragments of SLC17As mutations in LUAD and LUSC. 
A

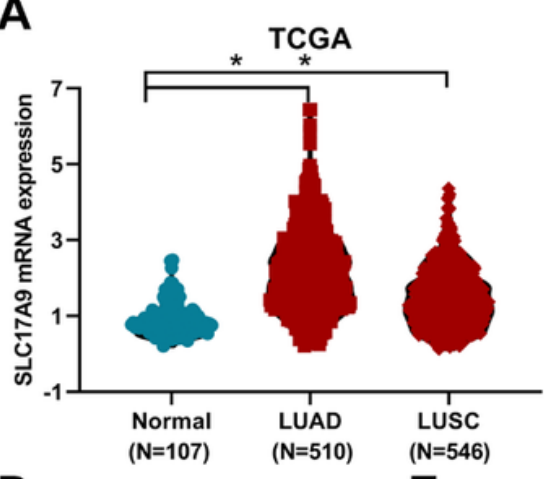

D

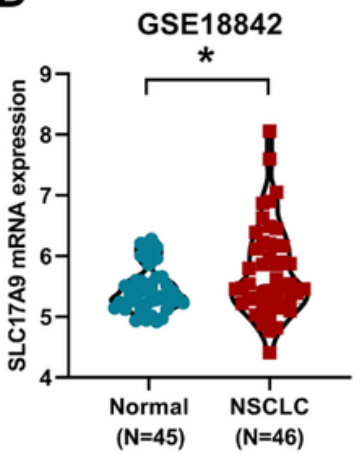

G

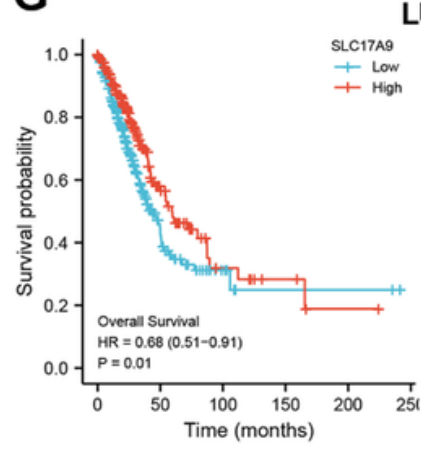

I

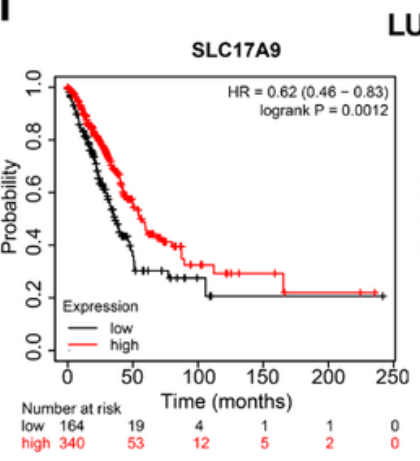

LUAD
B

E

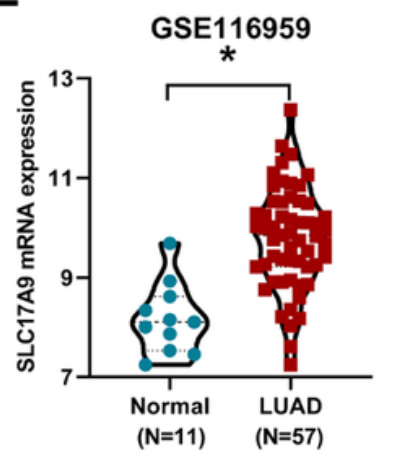

GSE74706

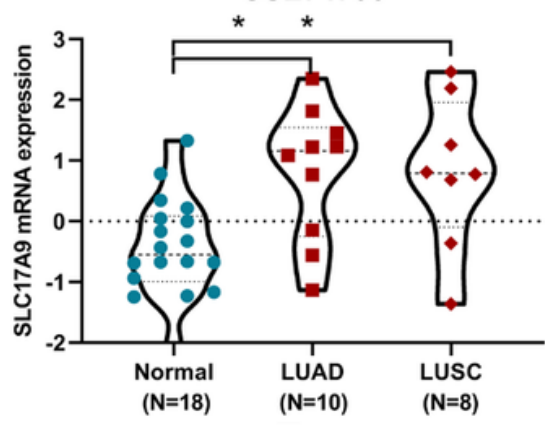

F
C

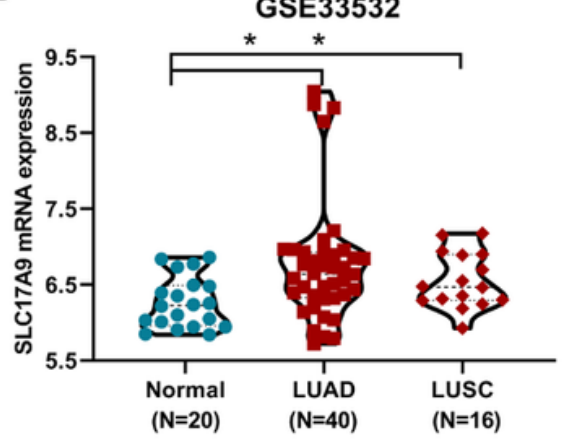

GSE4127

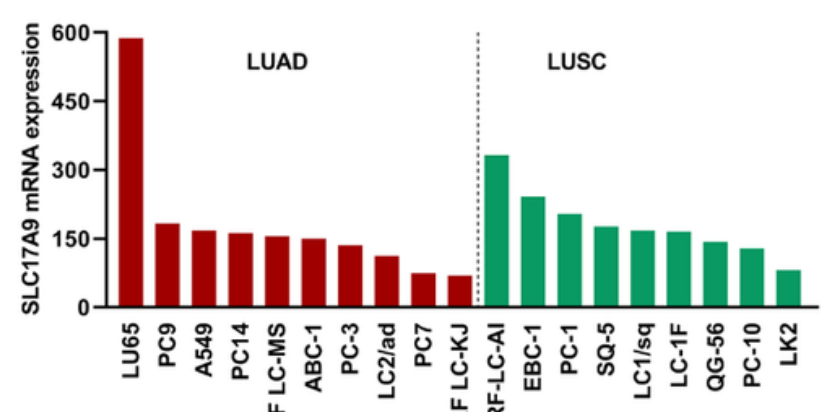

H
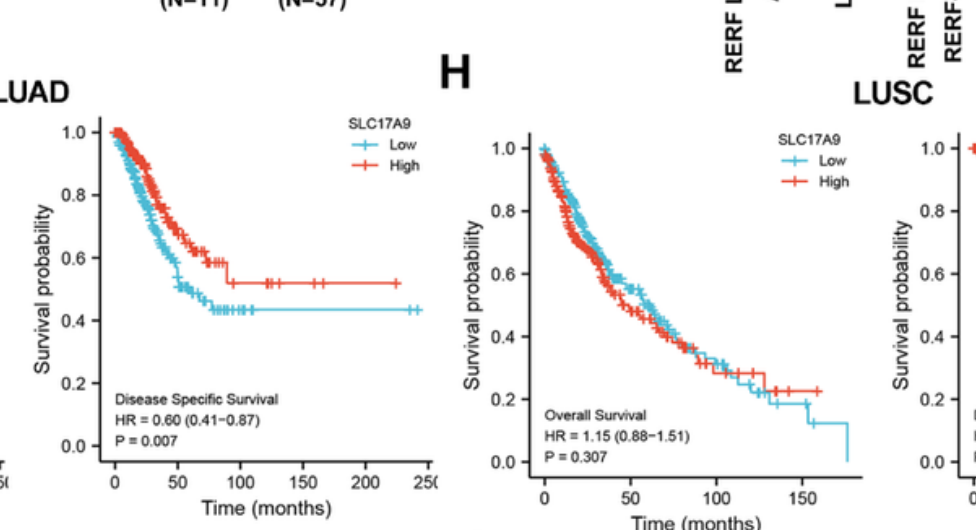

LUSC
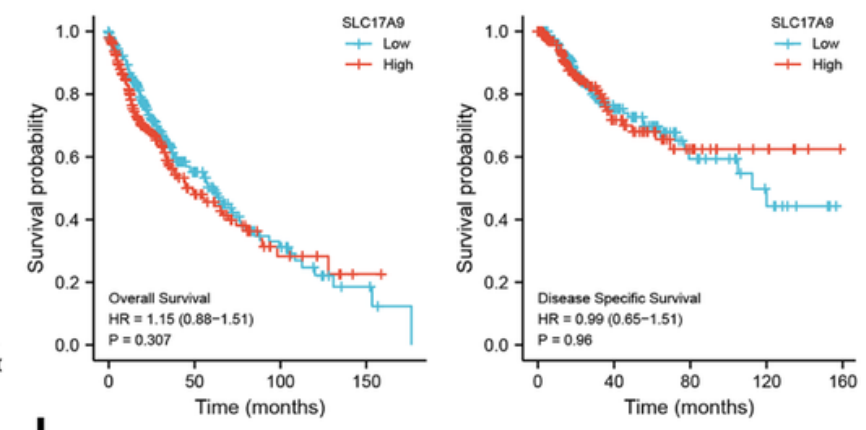

J

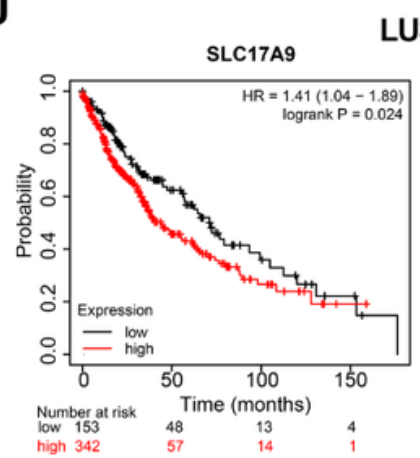

LUSC
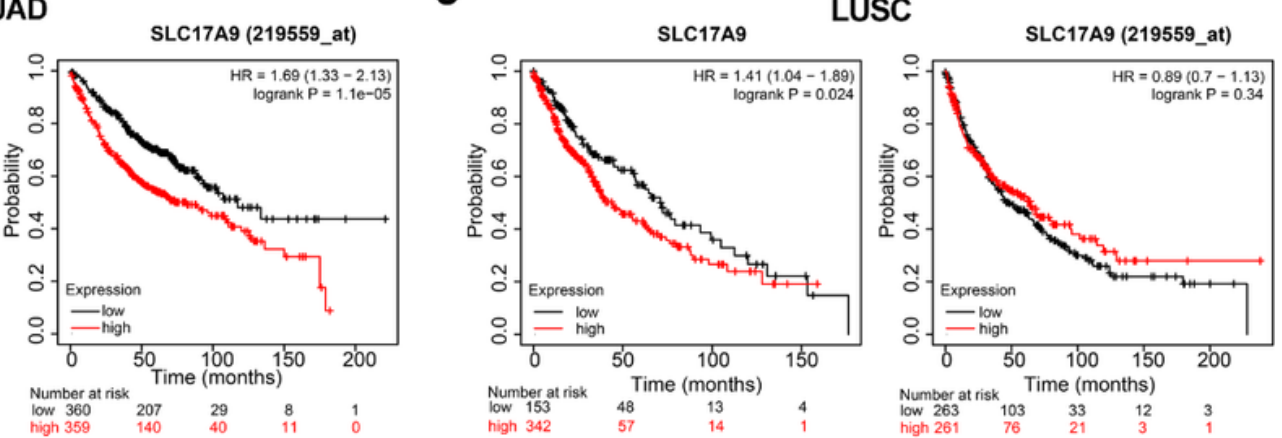

Figure 4

Bioinformatics analysis of the expression pattern and overall survival (OS) rates of SLC17A9 in NSCLC and normal tissues. (A) The mRNA expression of SLC17A9 in tumor tissues and normal tissues based on the data from TCGA-LUAD and LUSC cohort, GSE74706 (B), GSE33532 (C), GSE18842 (D), GSE116959 (E) , *P< 0.05. $(F)$ The data of SLC17A9 mRNA in different LUAD and LUSC cell lines in GEO cohort (GSE4127), *P $<0.05$. (G I) OS analysis according to mRNA expression of SLC17A9 in LUAD and LUSC $(\mathrm{H} \mathrm{J})$ patients using KaplanMeier plotter and R package. 
A

LUAD

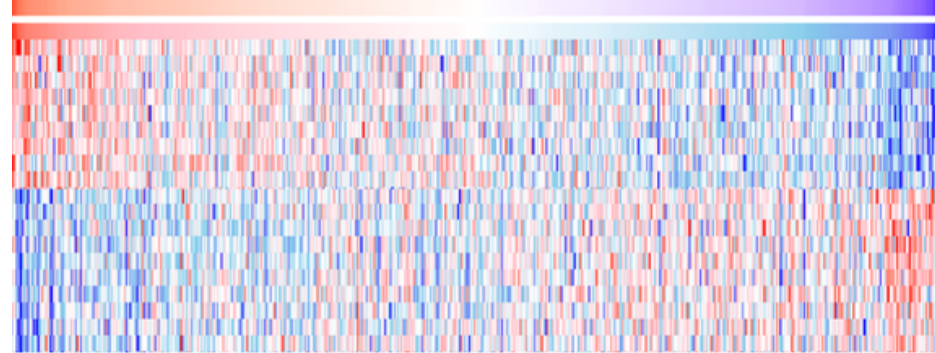

LUSC

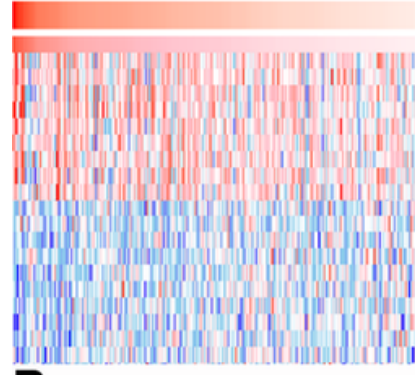

B

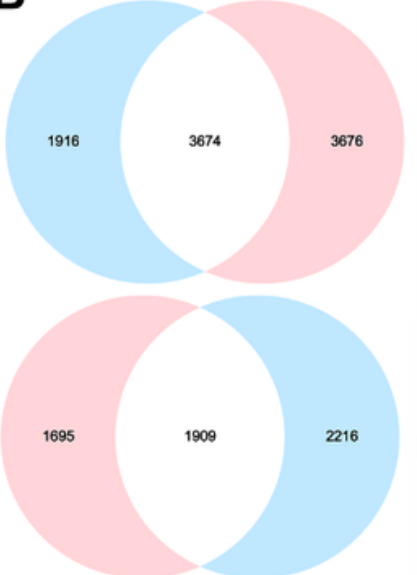

ID-77736 LUAD ID-78789 LUSC

E

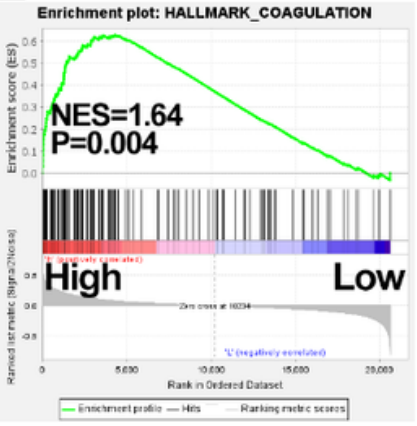

D

$\mathbf{F}$

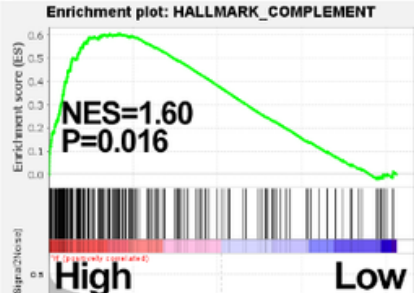

C GO enriched terms

\section{biological regulation}

melolic process

response to stimulus

multicellular organismal process

localization

communication

cellular compontal process

multi-organism process

cell proliferation membrane

membrane-enclosed lumen protein-containing complex

cytosol cytosol vesicle extracellular space cell projection ion binding

nucleic acid binding hydrolase activity transferase activity nucleotide binding $\begin{array}{r}\text { transporter activity } \\ \text { enzyme regulator activity }\end{array}$
structural molecule activity

KEGG enriched terms

$\begin{array}{llllll}-0.5 & 0.0 & 0.5 & 1.0 & 1.5 & 2.0\end{array}$

Biological Process

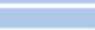

Cellular Component
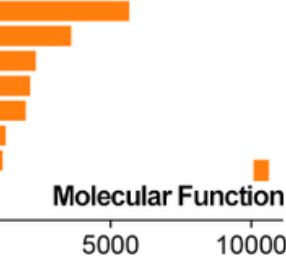
rotein binding molecular transducer activity

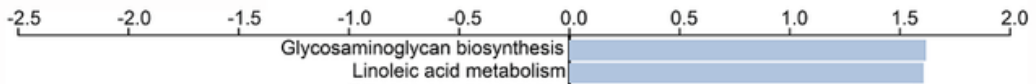

Other types of glycan degradation

Taste transduction
Ther types of O-glycan biosynthesis

Staphylococcus aureus infectio

Protein digestion and absorption

Primary immunodeficiency

Phospholipase D signaling pathway

Fructose and mannose metabolism African trypanosomiasis
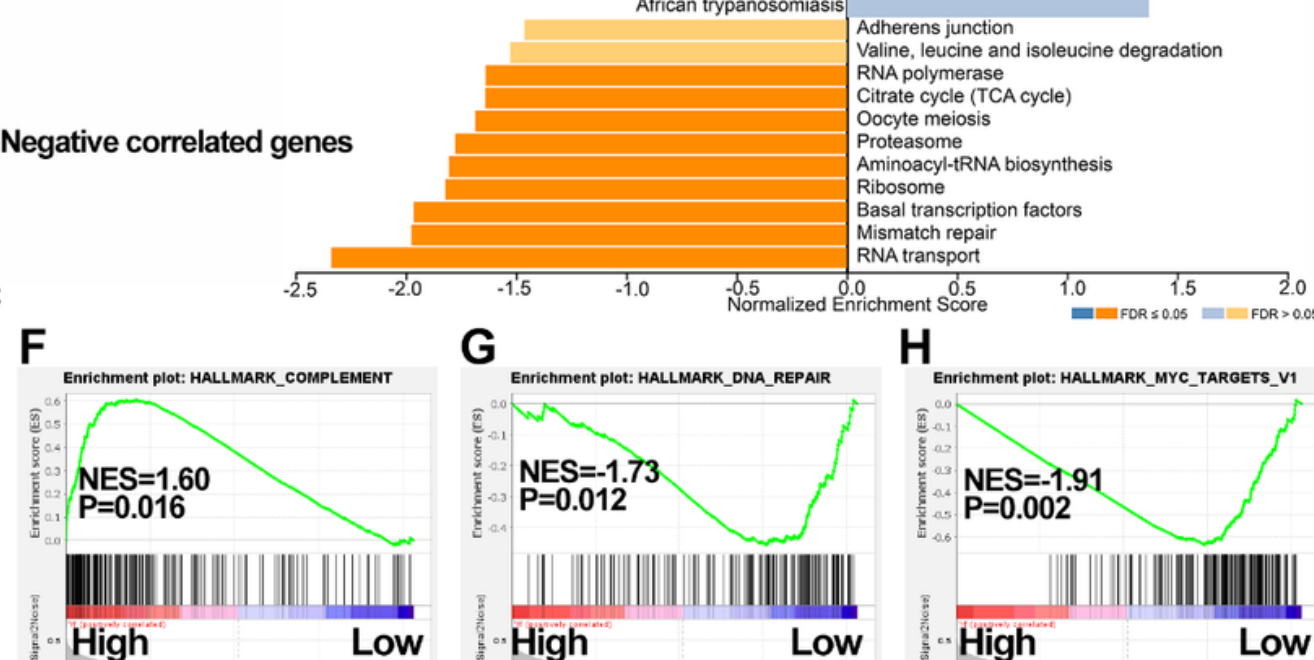

$\mathbf{H}$

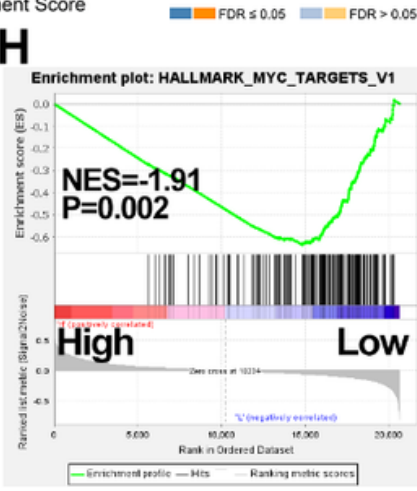

\section{Figure 5}

The related signaling pathways associated with SLC17A9 expression levels identified by LinkedOmics and GSEA. (A) Heat maps showing top 10 genes positively (red) and negatively (blue) correlated with SLC17A9 in LUAD and LUSC in LinkedOmics. (B) Venn's diagram of positive or negative correlated genes of SLC17A9 in LUAD and LUSC. (C) The most significantly enriched GO annotations and KEGG pathways (D) of SLC17A9 identified by comparing LUAD and LUSC. (E) Strong correlation between SLC17A9 and coagulation, 
complement (F), DNA repair (G) and myc targets (H) in the GSEA/Hallmark category (GSE18842). ES, enrichment score; NES, normalized ES; NOM p-val, normalized p-value.
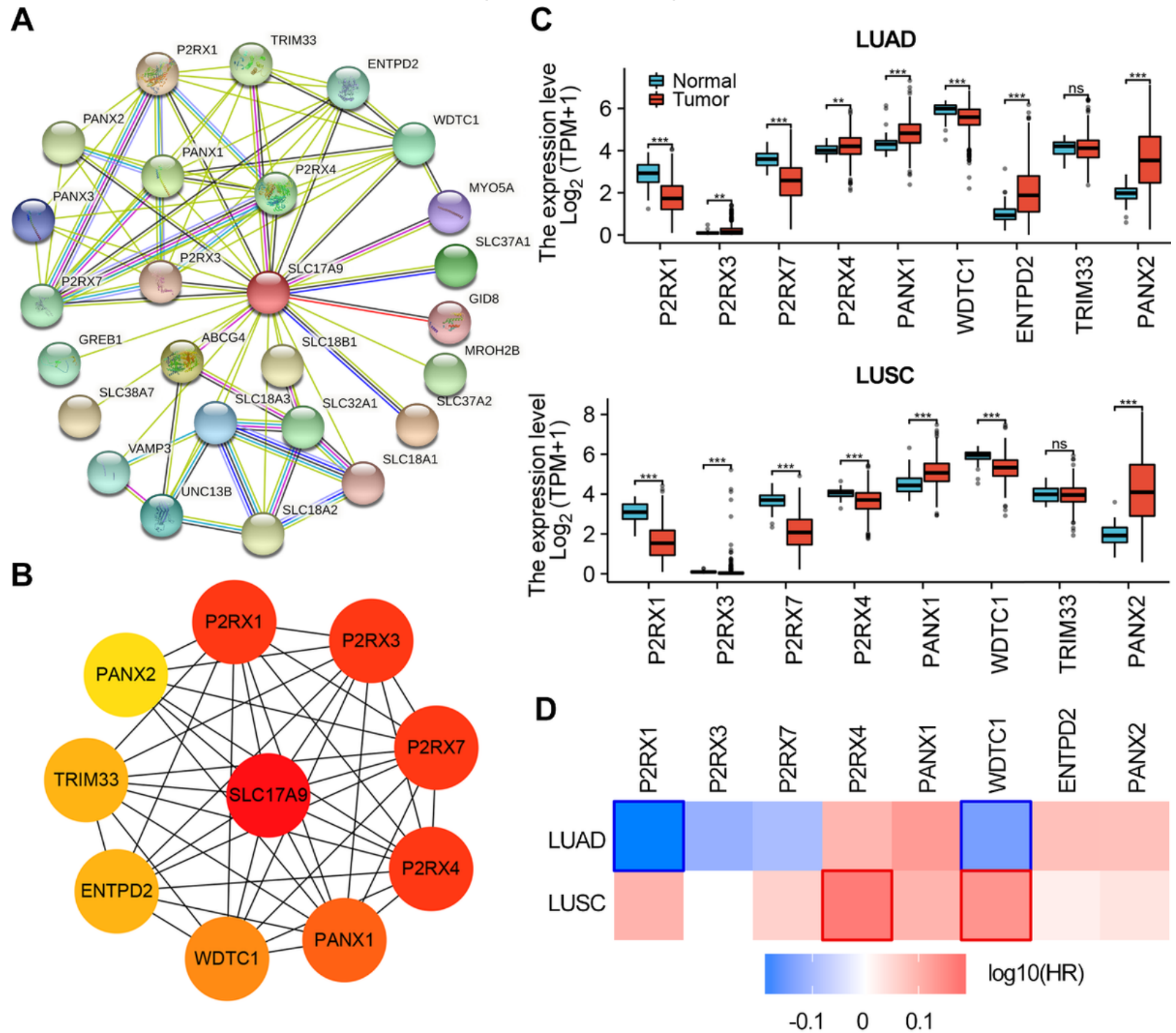

Figure 6

Protein-protein interaction (PPI) network constructed by STRING database for SLC17A9-related gene. (A) PPI network. (B) Ten hub genes in the PPI screened using the cytoHubba module of Cytoscape (higher color represents stronger connectivity). (C) Expression levels of the hub genes based on TCGA data. (D) Overall survival map of hub genes in LUAD and LUSC by GEPIA2. 

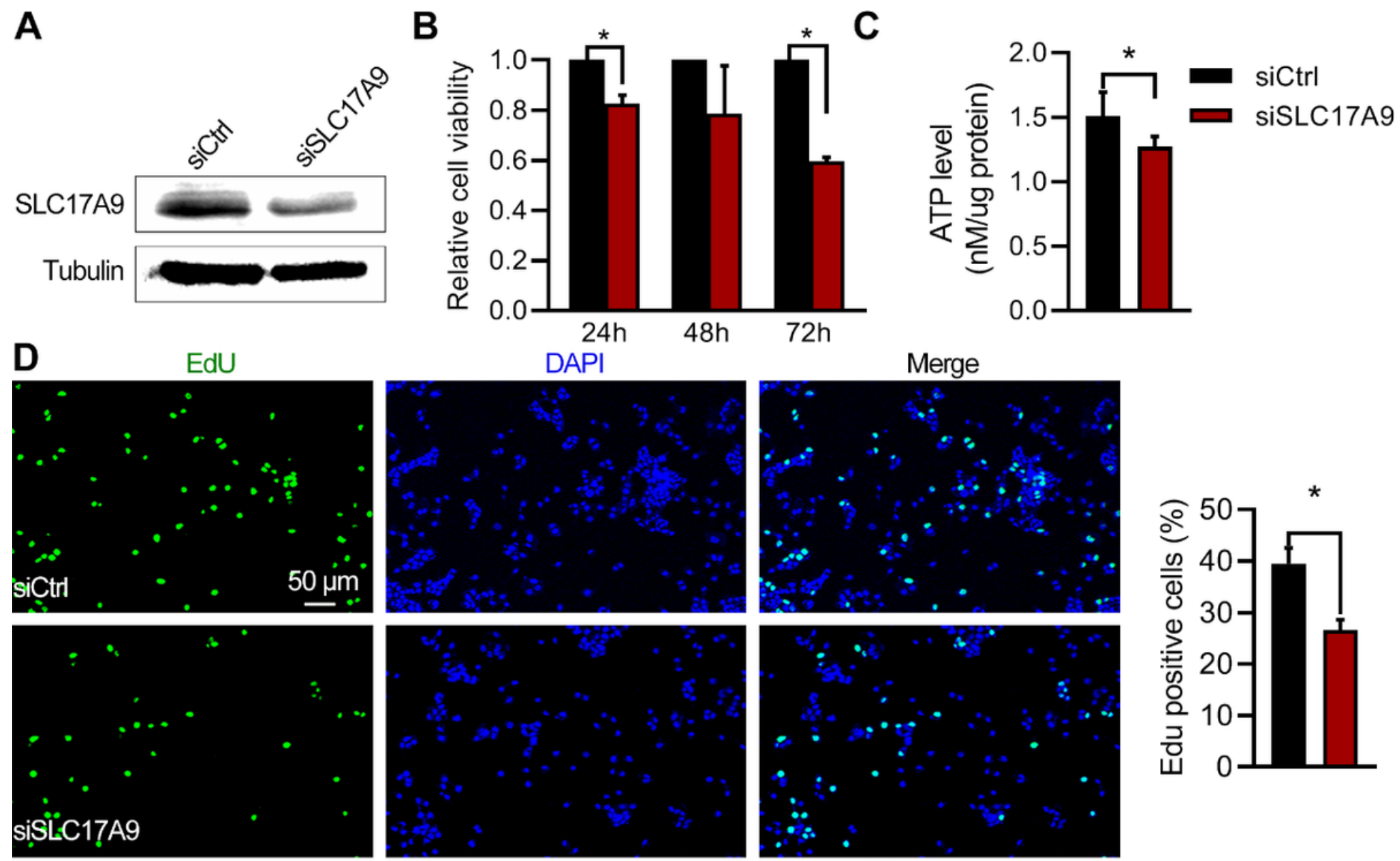

$\mathbf{E}$
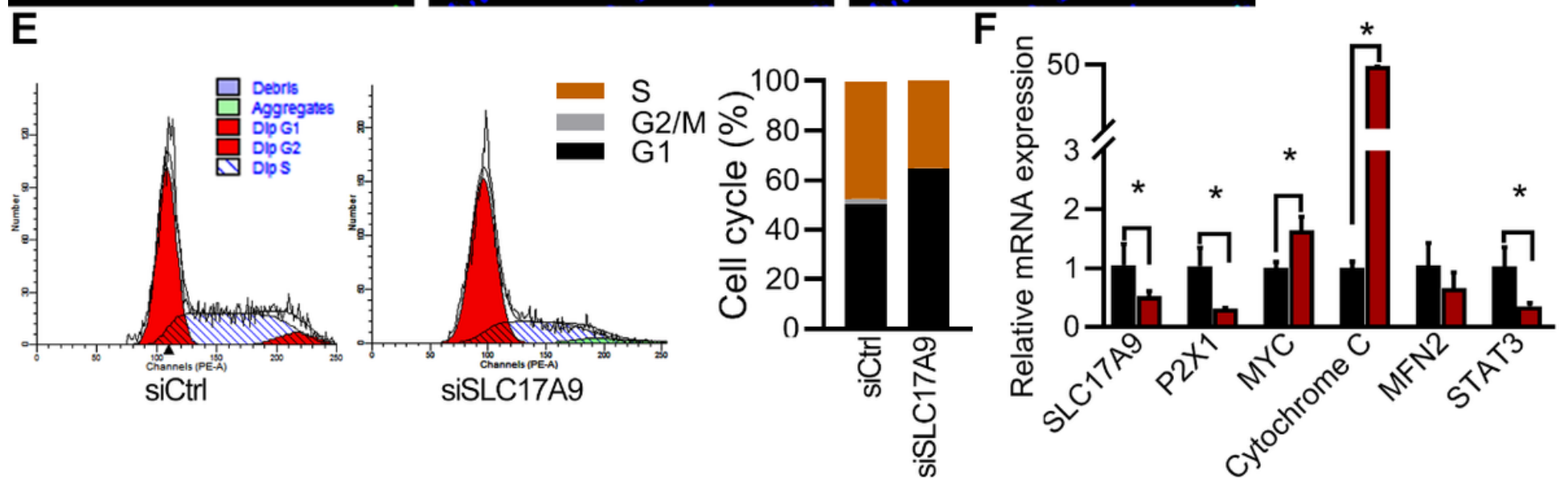

Figure 7

Downregulation of SLC17A9 inhibited cell proliferation through upregulation of MYC and cytochrome C. (A) Western blots showed the knockdown efficacy of SLC17A9 siRNA. (B) Cell growth was determined by MTS assay. (C) ATP levels in A549 cells after $48 \mathrm{~h}$ of siRNA treatment. (D) Proliferation rate was quantified by EdU assay. (E) Cell cycle distribution analysis by flow cytometry in A549 cells transfected with SLC17A9 siRNA or control oligos. (F) Real time PCR analysis of SLC17A9, P2X1, MYC, cytochrome C, MFN2, and STAT3 in SLC17A9 siRNA transfected cells or control cells. 
A

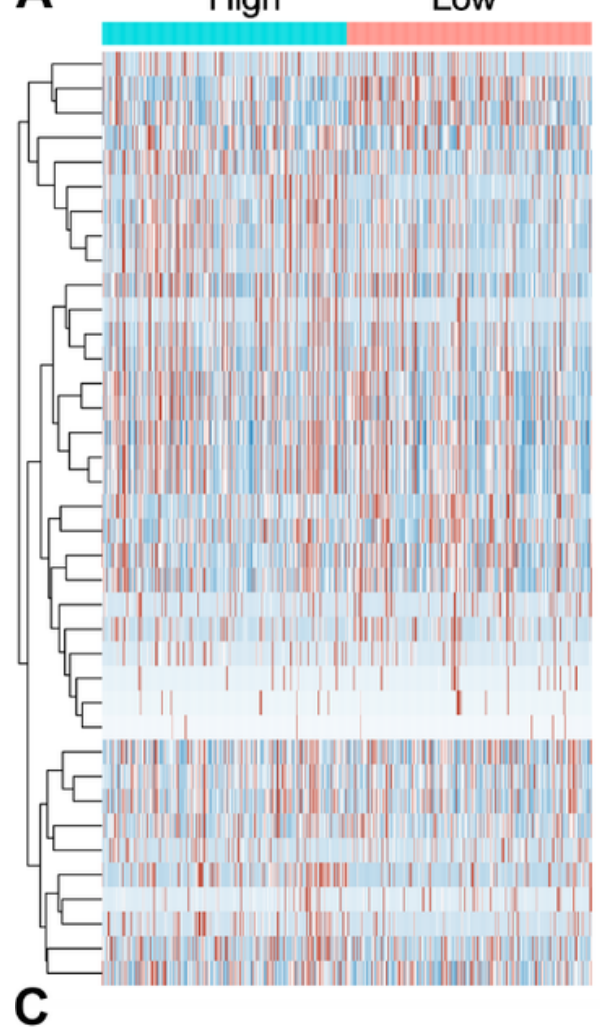

Groups

T cell CD8+ naive ${ }^{\star}$

Common lymphoid progenitor**

T cell CD4+ Th2 ${ }^{\star \star}$

T cell CD4+ Th1

B cell plasma ${ }^{* *}$

Class-switched memory B cell ${ }^{\star \star *}$

B cell memory ${ }^{\star \star \star}$

Plasmacytoid dendritic cell

T cell CD8+ effector memory

T cell CD8+*

T cell CD8+ central memory

Macrophage

Macrophage M1*

Myeloid dendritic cell activated

immune score

microenvironment score *

Granulocy

Mast cell

Myeloid dendritic cell

Monocyte

T cell regulatory (Tregs)

T cell CD4+ memory

$B$ cell naive

T cell CD4+ (non-regulatory) NK cell

T cell gamma delta

Hematopoietic stem cell

Endothelial cell

stroma score

Macrophage M2

Neutrophil

T cell CD4+ central memory

Common myeloid progenitor

Eosinophil

T cell CD4+ effector memory* $\mathrm{T}$ cell NK

B

$2.24 \mathrm{e}-02$

$2.71 \mathrm{e}-09$

$1.67 \mathrm{e}-01$

$1.43 \mathrm{e}-03$

$2.05 \mathrm{e}-03$

$2.05 e-03$
$6.16 e-04$

$6.16 \mathrm{e}-04$
$4.09 \mathrm{e}-04$

$2.58 \mathrm{e}-03$

$1.38 \mathrm{e}-01$

$3.94 \mathrm{e}-01 \quad-1$

$3.73 \mathrm{e}-02$

$6.55 \mathrm{e}-01$

$1.80 \mathrm{e}-01 \quad-2$

$2.77 \mathrm{e}-02$

$4.68 \mathrm{e}-01$

$5.13 \mathrm{e}-02$

$4.99 \mathrm{e}-02$

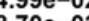

$8.24 \mathrm{e}-01$

$8.24 \mathrm{e}-01$
$3.01 \mathrm{e}-01$

$3.01 \mathrm{e}-01$
$9.65 \mathrm{e}-01$

$4.08 \mathrm{e}-01$

$2.69 \mathrm{e}-02$

$7.62 \mathrm{e}-02$

$7.69 \mathrm{e}-03$

$7.69 \mathrm{e}-03$
$4.92 \mathrm{e}-01$

$4.92 \mathrm{e}-01$
$2.02 \mathrm{e}$

$2.02 \mathrm{e}-01$
$8.44 \mathrm{e}-01$

$6.53 \mathrm{e}-02$

$5.73 \mathrm{e}-02$

$5.54 \mathrm{e}-01$

$9.98 \mathrm{e}-02$

9.98e-02

4.81e-01

$4.81 \mathrm{e}-01$
$1.06 \mathrm{e}-04$

$2.71 \mathrm{e}-02$

$1.34 \mathrm{e}-01$

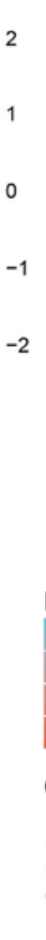

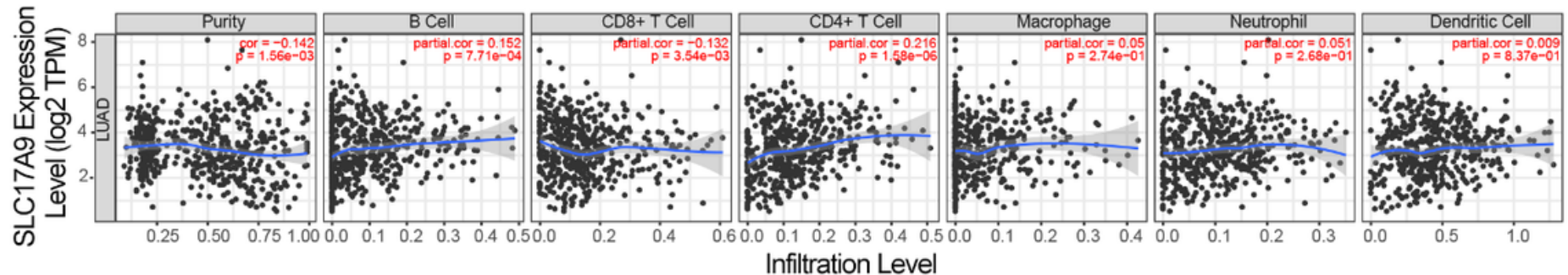

D nfiltration Leve

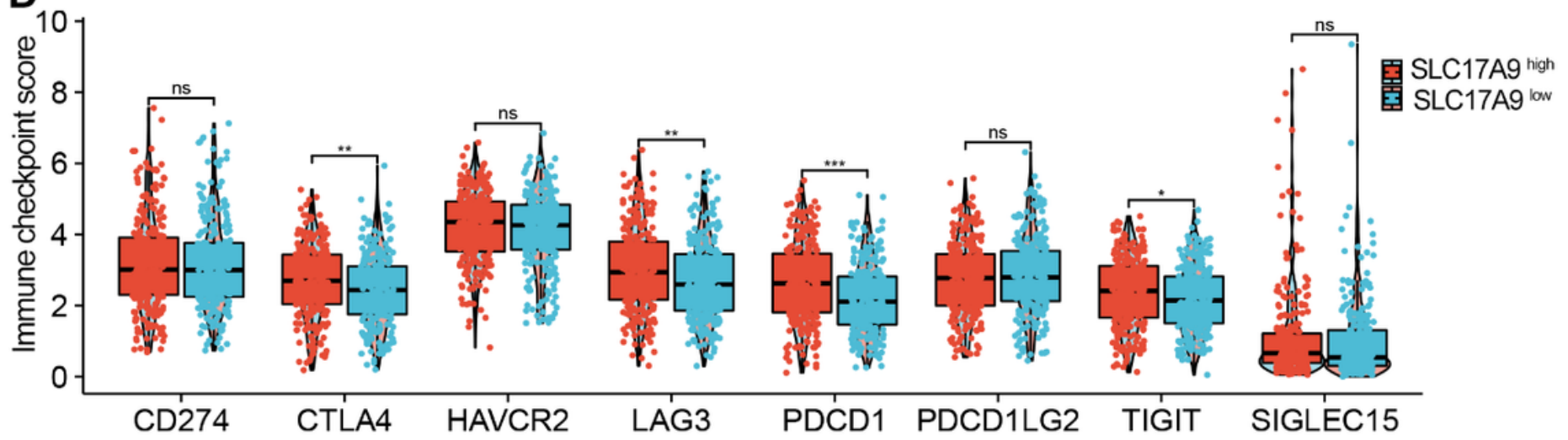

Figure 8

Correlation analysis of SLC17A9 expression with immune infltration level in LUAD. (A) Heatmap of relationship between tumor infiltrating immune cells and SLC17A9 expression levels in each sample, *p < $0.05,{ }^{* *} p<0.01,{ }^{* *} p<0.001$. (B) Lollipop diagram and bar charts showing association between tumor infiltrating immune cells for SLC17A9 expression status. (C) The correlation between SLC17A9 expression levels and the tumor-infiltration in TIMER database. (D) Violin plot of immune checkpoints related genes in TCGA-LUAD tumor tissues with high or low SLC17A9 expression. 
A

High

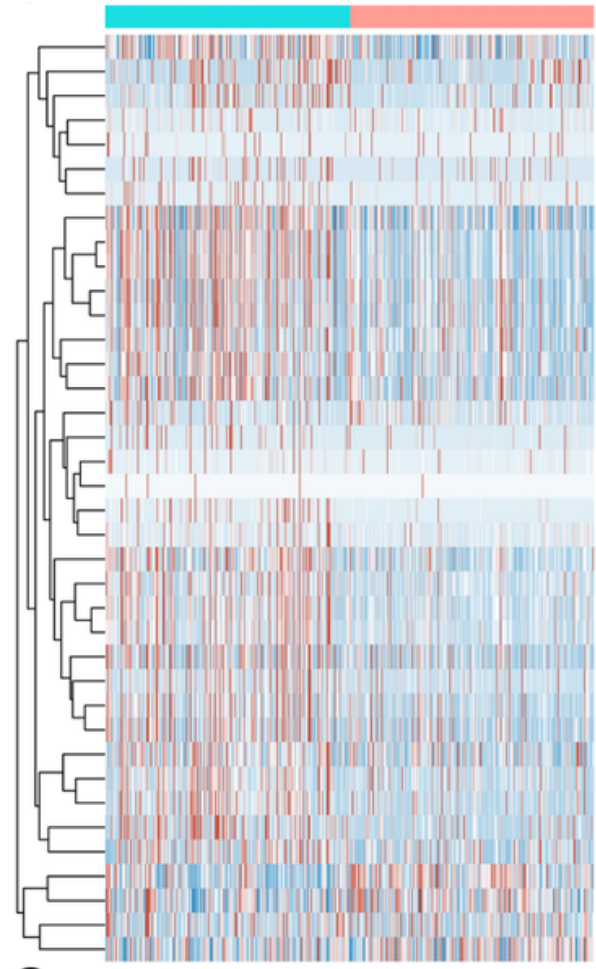

Groups

Mast cell ${ }^{\star \star *}$

T cell CD4+ central memory

Eosinophil*

Neutrophil

NK cell

Common myeloid progenitor*

Granulocyte-monocyte progenitor

Myeloid dendritic cell activated ${ }^{\star \star \star} \quad 3.21 \mathrm{e}-01$

mmune score ${ }^{\star \star \star \star}$

microenvironment score ${ }^{\star \star *}$

Macrophage

Macrophage M1*ะ

Macrophage M2**

Myeloid dendritic cell ${ }^{\star \star \star *}$

Monocyte

T cell CD4+ memory

T cell regulatory (Tregs)*

$T$ cell $\mathrm{CD} 4+$ (non-regulatory) ${ }^{*}$

T cell gamma delta

$T$ cell CD4+ naive**

B cell naive ${ }^{\star}$

B cell plasma**

Class-switched memory B cell

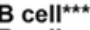

B cell memory ***

Plasmacytoid dendritic cell

T cell CD8+ effector memory

T cell CD8+**

$T$ cell CD8+ central memory ${ }^{\star \star \star}$

Hematopoietic stem cell ${ }^{\star \star \star}$

Endothelial cell ${ }^{* \star}$

stroma score

T cell CD4+ effector memory **

T cell NK $^{\star \star \star}$

Common lymphoid progenitor

T cell CD4+ Th2 ${ }^{*+}$

$\mathrm{T}$ cell CD8+ naive

$T$ cell CD4+ Th1 ${ }^{\star}$

C

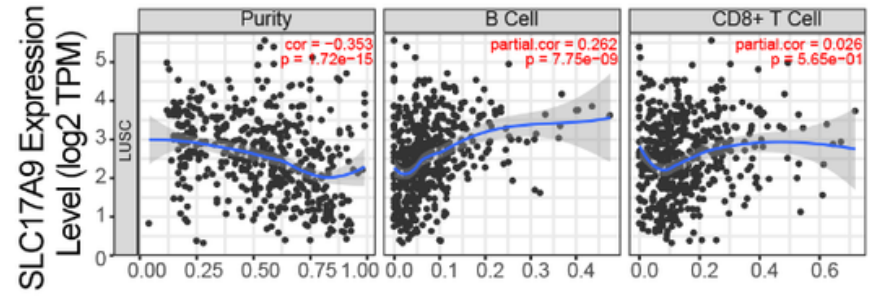

D

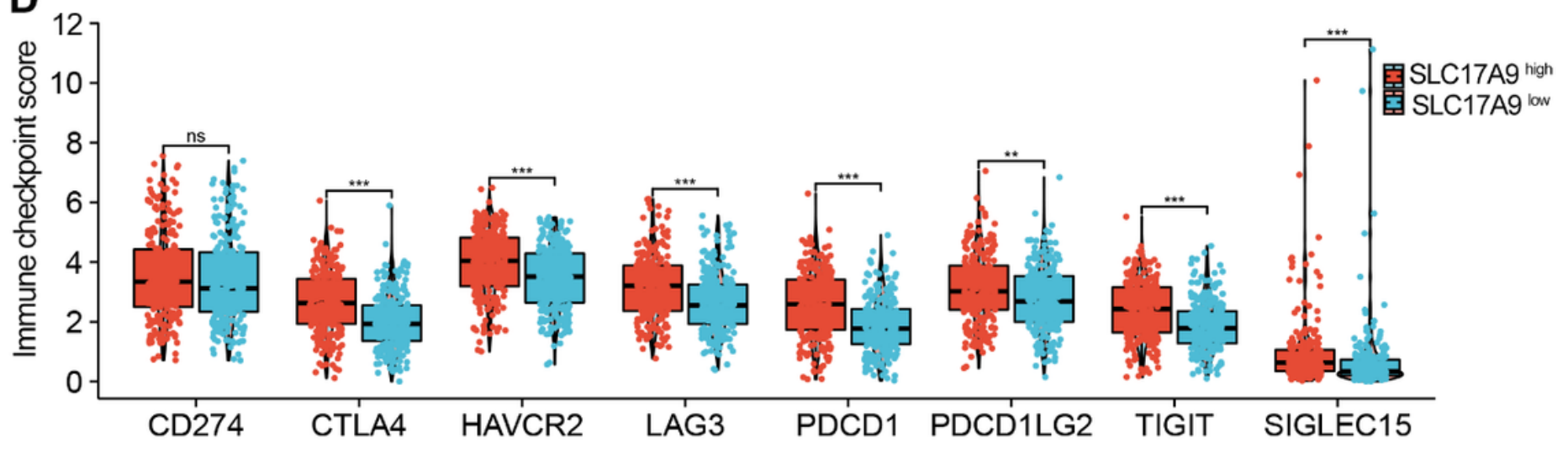

Figure 9

The landscape of immune infiltration draw by SLC17A9 expression in LUSC. (A) Heatmap and lollipop diagram (B) showing the relationship between tumor infiltrating immune cells and SLC17A9 expression levels in each sample, ${ }^{\star} p<0.05,{ }^{*} p<0.01,{ }^{\star *} p<0.001$. (C) The correlation analysis from TIMER database. (D) Expression of immune checkpoints related genes in TCGA-LUSC tumor tissues with high or low SLC17A9 expression.
B

$5.93 \mathrm{e}-07$
$3.10 \mathrm{e}-03$

$7.82 \mathrm{e}-07$

$1.68 \mathrm{e}-06$
$9.57 \mathrm{e}-01$

$4.02 \mathrm{e}-02$

$3.76 \mathrm{e}-02$

$2.18 \mathrm{e}-01$

$1.81 \mathrm{e}-04$

$1.39 \mathrm{e}-09$

$1.01 \mathrm{e}-06$

$2.95 \mathrm{e}-11$

$1.80 \mathrm{e}-08$

$2.96 \mathrm{e}-04$

$6.11 \mathrm{e}-09$

.58e-06

$2.87 \mathrm{e}-04$

$3.40 \mathrm{e}-07$
$6.47 \mathrm{e}-13$

$2.11 \mathrm{e}-08$

$1.89 \mathrm{e}-04$

$1.54 \mathrm{e}-10$

$2.54 \mathrm{e}-03$

$3.80 \mathrm{e}-01$

$1.83 \mathrm{e}-02$
Correlation

ㅇ 0.1

O 0.2

○े 0.3

NK CD56dim cells

NK CD56bright cells

$T$ helper cells

Mast cells

iDC

Neutrophils

Th17 cells

Th2 cells

$\begin{array}{lllllllllllll}0.0 & 0.1 & 0.2 & 0.3 & 0.4\end{array}$

Correlation

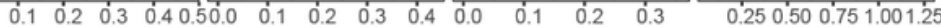
Infiltration Level

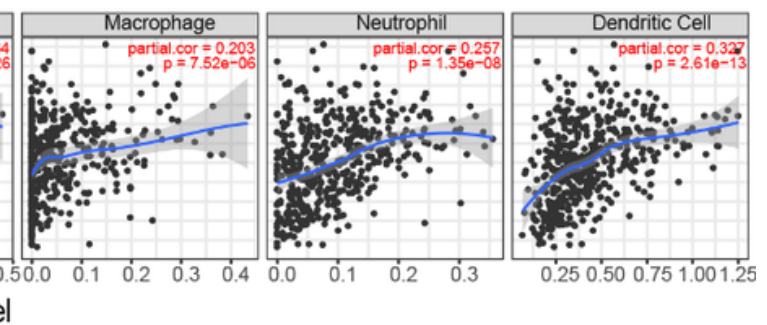

